\section{RSP}

http://www.rsp.fsp.usp.br/
Revista de Saúde Pública

\title{
Evolução da ingestão de energia e nutrientes no Brasil entre 2008-2009 e 2017-2018
}

\author{
Eliseu Verly Junior' (iD, Dirce Maria Marchioni"l' iD, Marina Campos Araujo" iD, Eduardo De \\ Carli"I' ID, Dayan Carvalho Ramos Salles de Oliveira' iD, Edna Massae Yokoo'v (iD, Rosely \\ Sichieri' (iD, Rosangela Alves Pereirav (iD \\ ' Universidade do Estado do Rio de Janeiro. Instituto de Medicina Social. Departamento de Epidemiologia. Rio \\ de Janeiro, RJ, Brasil \\ " Fundação Oswaldo Cruz. Escola Nacional de Saúde Pública Sérgio Arouca. Departamento de Epidemiologia e \\ Métodos Quantitativos em Saúde. Rio de Janeiro, RJ, Brasil \\ II' Universidade de São Paulo. Faculdade de Saúde Pública. Departamento de Nutrição. São Paulo, SP, Brasil \\ iv Universidade Federal Fluminense. Instituto de Saúde Coletiva. Departamento de Epidemiologia e Bioestatística. \\ Niterói, RJ, Brasil \\ $\checkmark$ Universidade Federal do Rio de Janeiro. Instituto de Nutrição Josué de Castro. Departamento de Nutrição \\ Social e Aplicada. Rio de Janeiro, RJ, Brasil
}

\author{
Correspondência: \\ Eliseu Verly Junior \\ Universidade do Estado do Rio de \\ Janeiro \\ Instituto de Medicina Social \\ Departamento de Epidemiologia \\ Rua São Francisco Xavier, 524 - \\ Bloco $\mathrm{E}, 7^{\circ}$ andar \\ 20550-013 Maracanã, RJ, Brasil \\ E-mail: eliseu.junior@uerj.br
}

Recebido: 18 nov 2020

Aprovado: 8 mai 2021

Como citar: Verly-Jr E, Marchioni DM, Araujo MC, De Carli E, Oliveira DCRS, Yokoo EM, et al. Evolução da ingestão de energia e nutrientes no Brasil entre 2008-2009 e 2017-2018. Rev Saude Publica. 2021;55 Supl 1:5s. https://doi.org/10.11606/s15188787.2021055003343

Copyright: Este é um artigo de acesso aberto distribuído sob os termos da Licença de Atribuição Creative Commons, que permite uso irrestrito, distribuição e reprodução em qualquer meio, desde que o autor e a fonte originais sejam creditados.

\section{RESUMO}

OBJETIVO: Avaliar a evolução da ingestão de energia e nutrientes e a prevalência de inadequação da ingestão de micronutrientes segundo características sociodemográficas e regiões brasileiras.

MÉTODOS: Foi analisado o consumo alimentar de 32.749 indivíduos do Inquérito Nacional de Alimentação da Pesquisa de Orçamentos Familiares de 2008-2009, por dois registros alimentares, e de 44.744 indivíduos a partir de dois recordatórios de 24 horas em 2017-2018. Estimaram-se a ingestão usual e o percentual de indivíduos com consumo abaixo da necessidade média para cálcio, magnésio, fósforo, cobre e zinco, vitaminas A, C, D, E, tiamina, riboflavina, piridoxina e cobalamina. A ingestão de sódio foi comparada ao valor de referência para reduzir risco de doenças crônicas. As análises foram estratificadas por sexo, faixa etária, região e renda.

RESULTADOS: A ingestão energética diária média foi de 1.753 kcal em 2008-2009 e 1.748 kcal em 2017-2018. As prevalências de inadequação mais elevadas (> 50\%) nos dois períodos foram de cálcio, magnésio, vitaminas A, D e E, piridoxina e, somente entre adolescentes, fósforo. Houve aumento na prevalência de inadequação de vitamina $\mathrm{A}$, riboflavina, cobalamina, magnésio e zinco entre as mulheres, e de riboflavina entre os homens. A prevalência de inadequação diminuiu para a tiamina. A ingestão de sódio foi excessiva em aproximadamente $50 \%$ da população nos dois períodos. As variações mais altas (cerca de 50\%) nas prevalências de inadequação entre os extremos de renda (< 0,5 salário-mínimo e $>2$ salários-mínimos per capita) foram observadas para vitamina B12 e C nos dois períodos. As regiões Norte e Nordeste apresentaram maiores prevalências de inadequação.

CONCLUSÃO: Ambos os inquéritos verificaram prevalências elevadas de inadequação de ingestão de nutrientes e consumo excessivo de sódio. A inadequação varia de acordo com os estratos de renda, aumentando nas regiões mais pobres do país.

DESCRITORES: Consumo de Alimentos. Ingestão de Energia. Deficiências Nutricionais, epidemiologia. Alimentos, Dieta e Nutrição. Inquéritos sobre Dietas. 


\section{INTRODUÇÃO}

O impacto de dietas de baixa qualidade na morbidade e mortalidade por doenças crônicas não transmissíveis (DCNT) em todo o mundo foi maior que o de qualquer outro fator de risco, incluindo tabagismo, segundo o Global Burden of Disease ${ }^{1}$. A má nutrição em países de baixa e média renda é caracterizada com frequência pelo excesso de ingestão de energia e deficiência de micronutrientes, tornando desafiador o enfrentamento da dupla carga de doenças geradas pela coexistência da obesidade e da desnutrição, além de outras doenças não transmissíveis relacionadas à dieta² .

A segurança alimentar e a nutrição estão intimamente ligadas. A insegurança alimentar se relaciona com a má qualidade da dieta, que por sua vez está ligada a manifestações de má nutrição que aumentam o risco de desnutrição, sobrepeso e obesidade 2 . Em 2015, os países das Nações Unidas, que incluem o Brasil, se comprometeram com os 17 Objetivos de Desenvolvimento Sustentável (ODS) da Agenda 2030. No ODS 2, a meta 2.2 é acabar com todas as formas de má nutrição³.

No Brasil, em 2019, 26\% da população maior de 18 anos estava obesa e cerca de $60 \%$ com sobrepeso ${ }^{4}$. Entre 2002-2003 e 2019, a proporção de obesos na população com 20 anos ou mais de idade mais do que dobrou, indo de $12,2 \%$ para $26,8 \%{ }^{4}$. Por outro lado, dados da Pesquisa de Orçamentos Familiares mostraram que, em 2017-2018, dos 68,9 milhões de domicílios no Brasil, 36,7\% (o equivalente a 25,3 milhões) estavam com algum grau de insegurança alimentar 5 .

Os efeitos benéficos de um padrão saudável de alimentação derivam de um efeito cumulativo e sinergético de nutrientes de várias fontes alimentares ${ }^{6}$. Vitaminas e minerais são essenciais para os seres humanos e têm um papel importante em uma miríade de ciclos metabólicos que auxiliam ações celulares. Esses efeitos incluem a saúde cardiovascular e óssea, bem como a estrutura e a função do sistema nervoso ${ }^{7,8}$

A análise da situação nutricional e do consumo alimentar de uma população pode seguir várias abordagens complementares, que abrangem a ingestão de nutrientes, grupos de alimentos e padrões da dieta. A avaliação da ingestão de nutrientes permite conhecer os grupos da população em que há risco de inadequação, tanto para planejar ações quanto para analisar políticas públicas já implementadas, como as de fortificação de alimentos. Recomendações para a ingestão de nutrientes almejam assegurar que a maior parte da população receba quantidades que supram as necessidades fisiológicas 9 .

No Inquérito Nacional de Alimentação (INA) realizado em 2008-2009 ${ }^{10}$, o primeiro a coletar dados de consumo alimentar individual no Brasil, prevalências elevadas de inadequação foram observadas para vitamina $E$, vitamina $D$, cálcio, vitamina $A$, magnésio e vitamina $\mathrm{C}$ em adolescentes, adultos e idosos de ambos os sexos. Tendo em vista esse resultado, o presente estudo tem como objetivo avaliar a evolução da ingestão de energia e nutrientes e a prevalência de inadequação da ingestão de micronutrientes entre adolescentes, adultos e idosos brasileiros segundo características sociodemográficas e regiões brasileiras.

\section{MÉTODOS}

Foram analisados dados de dois Inquéritos Nacionais de Alimentação (INA), conduzidos em 2008-2009 e 2017-2018 como módulos da Pesquisa de Orçamentos Familiares (POF). Nos dois inquéritos, a amostragem foi definida por conglomerados, em dois estágios: no primeiro, foram sorteados os setores censitários; no segundo, os domicílios dentro de cada setor. Detalhes sobre o plano amostral são encontrados em outras publicações ${ }^{10,11}$.

As amostras dos INA corresponderam a $24,2 \%$ e $34,7 \%$ dos domicílios amostrados para a POF, totalizando 13.569 domicílios e 34.003 indivíduos em 2008-2009, e 20.112 domicílios e 46.164 indivíduos em 2017-2018. Todos os indivíduos com 10 anos ou mais de idade nos 
domicílios selecionados foram convidados a participar do módulo de consumo individual. Gestantes e lactantes não foram consideradas nessas análises ( $\mathrm{n}=1.254$ em 2008-2009; $\mathrm{n}=1.420$ em 2017-2018), totalizando 32.749 e 44.744 indivíduos no primeiro e no segundo inquérito, respectivamente.

\section{Coleta e Tratamento dos Dados}

No inquérito de 2008-2009, os dados de consumo alimentar foram obtidos por meio de registro alimentar preenchido pelo morador em dois dias não consecutivos (97\% responderam ao segundo registro). Os indivíduos foram orientados a anotar com detalhes todos os alimentos e bebidas consumidos, incluindo o modo de preparo, ingredientes e quantidades, preferencialmente em medidas caseiras. Ainda nos domicílios, os agentes de pesquisa passaram as informações de consumo alimentar para programa específico desenvolvido pelo Instituto Brasileiro de Geografia e Estatística ${ }^{11}$.

No inquérito de 2017-2018, a coleta foi feita por meio de recordatórios de 24 horas (R24h) aplicados em dois dias não consecutivos, na mesma semana em que os dados de despesa familiar foram obtidos (84\% responderam ao segundo R24h). Os indivíduos foram orientados a relatar todos os alimentos e bebidas consumidos no dia anterior às duas entrevistas, incluindo descrição detalhada dos alimentos (ingredientes, adições, modo de preparo) e quantidades. As entrevistas foram estruturadas segundo o método automatizado de múltiplas passagens ${ }^{12}$, utilizando para a coleta de dados um software especificamente elaborado para o inquérito, com 12 opções de itens que poderiam ser adicionados aos alimentos (azeite, manteiga, margarina, maionese, queijo, creme de leite, açúcar, mel, melado, ketchup, mostarda e molho shoyu).

Para analisar a quantidade e a composição nutricional das adições, considerou-se o tipo de adições reportadas. Adições à base de gordura (azeite, manteiga/margarina, maionese, queijo e creme de leite), quando relatadas, poderiam acrescentar no máximo $20 \%$ ao consumo, em gramas, do alimento ao qual foram adicionadas. As adições de açúcar, mel, melado, ketchup, mostarda e molho shoyu representaram no máximo $10 \%$ do consumo do item. Ou seja, se a um sanduíche foi adicionado ketchup e mostarda, cada adição representa $5 \%$ da gramatura do sanduíche. Detalhes sobre coleta dos dados, treinamento, procedimentos de crítica e imputação podem ser obtidos na publicação oficial ${ }^{10}$.

\section{Composição Nutricional dos Alimentos}

Para conversão dos alimentos relatados em quantidades de energia e nutrientes, em ambos os inquéritos utilizou-se a versão 7.0 da Tabela Brasileira de Composição de Alimentos (TBCA), elaborada pela Rede Brasileira de Dados de Composição de Alimentos, Universidade de São Paulo e Centro de Pesquisa em Alimentos ${ }^{13}$. É importante mencionar, porém, que a tabela de composição nutricional mudou entre os inquéritos. A justificativa dessa mudança se deve ao fato de a TBCA descrever dados nutricionais de alimentos brasileiros com a confiabilidade assegurada pela Rede Internacional de Sistema de Dados de Alimentos, da Organização das Nações Unidas para a Alimentação e a Agricultura (FAO), que determina diretrizes e critérios a serem usados na geração, compilação e utilização de dados de composição de alimentos ${ }^{14}$. Assim, para reduzir a possibilidade de divergências nas avaliações de tendência e permitir comparabilidade, a TBCA foi utilizada nas estimativas de energia e nutrientes dos dois inquéritos.

\section{Análise Estatística}

A distribuição da ingestão usual de cada nutriente foi estimada pelo método do National Cancer Institute (NCI) que aplica um modelo de regressão de efeito misto após a transformação Box-Cox dos dados de consumo ${ }^{15}$. São estimados parâmetros (média populacional, variâncias inter e intrapessoal, efeitos das variáveis grupo etário e sexo, renda e região e o lambda da transformação Box-Cox) que são posteriormente utilizados para construir as distribuições de consumo usual por meio de simulação de Monte Carlo. 
As distribuições da ingestão usual foram estimadas para cada grupo etário e sexo considerando as recomendações de ingestão de nutrientes definidas. As prevalências de inadequação foram estimadas utilizando o método da EAR (estimated average requirement) como ponto de corte, que representa o percentual de indivíduos com ingestão abaixo do valor médio estimado de necessidade para cada grupo etário e sexo ${ }^{16}$.

A ingestão de sódio foi comparada ao valor de referência para redução de risco de doenças crônicas (chronic disease reduction risk intakes), como proposto na última revisão dos valores de referência para sódio do Instituto de Medicina dos Estados Unidos ${ }^{17}$. A partir dessa referência calcula-se o percentual da população que deveria diminuir o consumo de sódio para reduzir o risco de doenças crônicas. Considerando a incompatibilidade entre os dados de niacina pré-formada, disponíveis na TBCA, com os valores de EAR expressos em equivalentes de niacina, prevalências de inadequação não foram calculadas para esse nutriente.

A prevalência de inadequação foi estimada para cada grupo etário e sexo para os quais há valores estabelecidos de necessidade média de ingestão ( 9 a 13 anos de idade, 14 a 18, 19 a 30, 31 a 50, 51 a 70, e 70 ou mais). Em seguida, foram calculadas as médias das prevalências ponderadas pelo tamanho amostral expandido de cada grupo etário, estratificadas em faixas etárias correspondentes aos ciclos de vida: adolescentes (10-18 anos), adultos (19-59 anos) e idosos (60 anos ou mais). O mesmo procedimento foi feito para totalizar prevalências por estratos de renda e região do país. Para os estratos de renda, utilizou-se o rendimento per capita das famílias: menor que 0,5, entre 0,5 e 1, entre 1 e 2, e maior que 2 salários-mínimos per capita. Os valores de salários-mínimos nas datas de referência das pesquisas foram: $\mathrm{R} \$ 415,00$ (15 de janeiro de 2009) e $\mathrm{R} \$ 954,00$ (15 de janeiro de 2018).

Os intervalos com 95\% de confiança (IC95\%) foram calculados para as médias e as prevalências de inadequação com base nos valores de erro-padrão corrigidos pelo desenho amostral, estimados pela técnica de replicação balanced repeated replication (BRR), usada no método do NCI. Utilizou-se o IC95\% para comparar as prevalências de inadequação entre sexos, faixas etárias, renda, regiões do país e os dois períodos avaliados. As análises foram executadas no software SAS versão 9.4.

\section{RESULTADOS}

As médias (IC 95\%) de ingestão energética diária se mostraram semelhantes nos dois inquéritos: $1.753 \mathrm{kcal}$ (1.734-1.772) em 2008-2009 e 1.748 kcal (1.732-1.763) em 2017-2018. Entre homens, a ingestão média calórica em 2008-2009, para adolescentes, adultos e idosos, foi de $1.996 \mathrm{kcal}$ (1.945-2.047), $1.969 \mathrm{kcal}(1.940-1.998)$ e $1.680 \mathrm{kcal}$ (1.633-1.726), respectivamente, e $1.969 \mathrm{kcal}$ (1.927-2.010), $2.018 \mathrm{kcal}$ (1.993-2.044) e $1.708 \mathrm{kcal}$ (1.669-1.747) em 2017-2018. Entre as mulheres, os valores foram de $1.753 \mathrm{kcal}$ (1.716-1.798), $1.549 \mathrm{kcal}$ (1.539-1.582) e $1.410 \mathrm{kcal}$ (1.370-1.449) em 2008-2009, e 1.674 kcal (1.633-1.715), 1.549 kcal (1.530-1.568) e $1.409 \mathrm{kcal}$ (1.381-1.437) em 2017-2018 para as mesmas faixas de idade, respectivamente.

Observa-se menor ingestão de energia entre mulheres e com o aumento da idade nos dois inquéritos, com exceção de homens adultos, cujas médias não diferiram da de adolescentes. Entretanto, em ambos os inquéritos, homens adultos, quando comparados às demais faixas etárias e às mulheres, tiveram as mais elevadas médias de ingestão para a maioria dos micronutrientes. Observou-se redução discreta $(<23 \%)$ entre os inquéritos nas médias de ingestão para a maioria dos micronutrientes, enquanto aumentos nas médias de ingestão (4\%-10\%) ocorreram somente entre adultos do sexo masculino para tiamina, e entre adultos de ambos os sexos para vitamina E (Tabela 1).

Prevalências de inadequação acima de $50 \%$ foram observadas para piridoxina, vitamina A e magnésio em todas as faixas etárias e, em específico, para fósforo entre adolescentes, tiamina entre adultos e idosos em ambos os inquéritos e, em 2017-2018, riboflavina entre homens idosos e adultos de ambos os sexos. Cálcio, vitamina D e vitamina E apresentaram as maiores inadequações em ambos os inquéritos (> 85\%) (Tabela 2). 
Tabela 1. Médias (IC95\%) de ingestão de vitaminas e minerais entre adolescentes, adultos e idosos nos Inquéritos Nacionais de Alimentação 2008-2009 e 2017-2018.

\begin{tabular}{|c|c|c|c|c|c|c|}
\hline \multirow{2}{*}{ Nutrientes } & \multicolumn{2}{|c|}{ Adolescentes } & \multicolumn{2}{|c|}{ Adultos } & \multicolumn{2}{|c|}{ Idosos } \\
\hline & 2008-2009 & 2017-2018 & 2008-2009 & 2017-2018 & 2008-2009 & 2017-2018 \\
\hline \multicolumn{7}{|l|}{ Homens } \\
\hline Cálcio (mg) & $\begin{array}{c}470.1 \\
(455.5-484,7)\end{array}$ & $\begin{array}{c}457,1 \\
(436,5-477,6)\end{array}$ & $\begin{array}{c}483,7 \\
(469,6-497,7)\end{array}$ & $\begin{array}{c}472,7 \\
(459,0-486,4)\end{array}$ & $\begin{array}{c}469,3 \\
(453,3-485,3)\end{array}$ & $\begin{array}{c}446,3 \\
(434,8-457,7)\end{array}$ \\
\hline Magnésio (mg) & $\begin{array}{c}285,8 \\
(280,3-291,2)\end{array}$ & $\begin{array}{c}273,3 \\
(266,6-280,0)\end{array}$ & $\begin{array}{c}310,0 \\
(307,2-312,8)\end{array}$ & $\begin{array}{c}304,7 \\
(301,5-307,9)\end{array}$ & $\begin{array}{c}285,3 \\
(277,6-293,0)\end{array}$ & $\begin{array}{c}275,7 \\
(270,6-280,8)\end{array}$ \\
\hline Fósforo (mg) & $\begin{array}{c}1.094,6 \\
(1.074,7-1.114,4)\end{array}$ & $\begin{array}{c}1.081,9 \\
(1.067,0-1.096,8)\end{array}$ & $\begin{array}{c}1.167,2 \\
(1.147,3-1.187,0)\end{array}$ & $\begin{array}{c}1.153,4 \\
(1.144,9-1.161,8)\end{array}$ & $\begin{array}{c}1.036,6 \\
(1.022,7-1.050,5)\end{array}$ & $\begin{array}{c}977,1 \\
(959,8-994,4)\end{array}$ \\
\hline Sódio (mg) & $\begin{array}{c}2.784,4 \\
(2.706,2-2.862,5)\end{array}$ & $\begin{array}{c}2.805,5 \\
(2.745,6-2.865,5)\end{array}$ & $\begin{array}{c}2.910,9 \\
(2.866,9-2.954,8)\end{array}$ & $\begin{array}{c}2.961,0 \\
(2.922,1-2.999,8)\end{array}$ & $\begin{array}{c}2.462,1 \\
(2.369,4-2.554,7)\end{array}$ & $\begin{array}{c}2.472,8 \\
(2.396,8-2.548,8)\end{array}$ \\
\hline Potássio (mg) & $\begin{array}{c}2.387,5 \\
(2.325,5-2.449,4)\end{array}$ & $\begin{array}{c}2.218,2 \\
(2.166,8-2.269,6)\end{array}$ & $\begin{array}{c}2.607,3 \\
(2.571,4-2.643,1)\end{array}$ & $\begin{array}{c}2.492,4 \\
(2.459,6-2.525,3)\end{array}$ & $\begin{array}{c}2.484,7 \\
(2.412,2-2.557,2)\end{array}$ & $\begin{array}{c}2.275,7 \\
(2.229,3-2.322,1)\end{array}$ \\
\hline Cobre (mg) & $1,4(1,4-1,5)$ & $1,4(1,4-1,5)$ & $1,6(1,5-1,6)$ & $1,6(1,5-1,6)$ & $1,4(1,3-1,5)$ & $1,4(1,4-1,4)$ \\
\hline Zinco (mg) & $11,8(11,3-12,2)$ & $11,5(11,1-11,9)$ & $12,9(12,7-13,1)$ & $12,7(12,5-12,8)$ & $11,5(11,2-11,9)$ & $10,9(10,8-11,0)$ \\
\hline Vitamina A (mcg) ${ }^{a}$ & $\begin{array}{c}376,3 \\
(343,2-409,3)\end{array}$ & $\begin{array}{c}324,6 \\
(307,6-341,7)\end{array}$ & $\begin{array}{c}378,5 \\
(359,3-397,8)\end{array}$ & $\begin{array}{c}320,1 \\
(306,6-333,6)\end{array}$ & $\begin{array}{c}376,5 \\
(357-396,0)\end{array}$ & $\begin{array}{c}342,4 \\
(324,7-360,0)\end{array}$ \\
\hline Tiamina (mg) & $1,0(1,0-1,1)$ & $1,1(1,1-1,2)$ & $1,0(0,9-1,0)$ & $1,1(1,1-1,1)$ & $0,9(0,8-0,9)$ & $0,9(0,9-1,0)$ \\
\hline Riboflavina (mg) & $1,3(1,3-1,4)$ & $1,2(1,2-1,3)$ & $1,2(1,2-1,3)$ & $1,1(1,1-1,1)$ & $1,2(1,2-1,2)$ & $1,1(1,1-1,1)$ \\
\hline Piridoxina (mg) & $0,8(0,8-0,8)$ & $0,8(0,8-0,9)$ & $0,8(0,7-0,8)$ & $0,8(0,8-0,8)$ & $0,6(0,6-0,7)$ & $0,7(0,7-0,7)$ \\
\hline Cobalamina (mcg) & $5,0(4,7-5,3)$ & $4,1(3,9-4,4)$ & $5,6(5,5-5,8)$ & $4,6(4,5-4,7)$ & $5,2(4,9-5,4)$ & $3,9(3,8-4,0)$ \\
\hline Vitamina D $(m c g)^{b}$ & $2,4(2,3-2,5)$ & $2,0(1,9-2,0)$ & $2,2(2,0-2,3)$ & $1,7(1,6-1,7)$ & $2,0(1,9-2,1)$ & $1,5(1,5-1,5)$ \\
\hline Vitamina $E(m g)^{c}$ & $6,6(6,4-6,8)$ & $6,7(6,6-6,9)$ & $7,0(6,9-7,0)$ & $7,3(7,2-7,5)$ & $6,2(6,1-6,4)$ & $6,4(6,3-6,6)$ \\
\hline Vitamina C (mg) & $\begin{array}{c}124,0 \\
(119,2-128,8)\end{array}$ & $\begin{array}{c}117,1 \\
(108,2-126,0)\end{array}$ & $\begin{array}{c}131,4 \\
(127,9-134,9)\end{array}$ & $\begin{array}{c}119,8 \\
(117,2-122,4)\end{array}$ & $\begin{array}{c}126,0 \\
(118,9-133,0)\end{array}$ & $\begin{array}{c}116,5 \\
(107,0-126,0)\end{array}$ \\
\hline Niacina $(m g)^{d}$ & $15,6(14,9-16,2)$ & $17,3(16,3-18,4)$ & $18,6(18,0-19,2)$ & $19,7(19,1-20,3)$ & $15,5(14,6-16,3)$ & $15,8(15,0-16,6)$ \\
\hline \multicolumn{7}{|l|}{ Mulheres } \\
\hline Cálcio (mg) & $\begin{array}{c}447,2 \\
(435,4-458,9)\end{array}$ & $\begin{array}{c}406,6 \\
(379,2-434,1)\end{array}$ & $\begin{array}{c}429,4 \\
(419,7-439,2)\end{array}$ & $\begin{array}{c}398,7 \\
(394,1-403,2)\end{array}$ & $\begin{array}{c}451,3 \\
(425,5-477,1)\end{array}$ & $\begin{array}{c}429,3 \\
(418,5-440,0)\end{array}$ \\
\hline Magnésio (mg) & $\begin{array}{c}244,3 \\
(238,9-249,7)\end{array}$ & $\begin{array}{c}229,7 \\
(224,2-235,2)\end{array}$ & $\begin{array}{c}240,8 \\
(238,9-242,7)\end{array}$ & $\begin{array}{c}232,8 \\
(229,1-236,6)\end{array}$ & $\begin{array}{c}232,4 \\
(229,9-234,9)\end{array}$ & $\begin{array}{c}225,5 \\
(221,9-229,1)\end{array}$ \\
\hline Fósforo (mg) & $\begin{array}{c}977,1 \\
(962,4-991,7)\end{array}$ & $\begin{array}{c}924,6 \\
(889,9-959,3)\end{array}$ & $\begin{array}{c}928,9 \\
(923,1-934,7)\end{array}$ & $\begin{array}{c}878,9 \\
(861,3-896,6)\end{array}$ & $\begin{array}{c}877,3 \\
(866,2-888,3)\end{array}$ & $\begin{array}{c}811,2 \\
(802,3-820,1)\end{array}$ \\
\hline Sódio (mg) & $\begin{array}{c}2.408,6 \\
(2.344,3-2.472,8)\end{array}$ & $\begin{array}{c}2.349,7 \\
(2.293,8-2.405,7)\end{array}$ & $\begin{array}{c}2.227 \\
(2.193,6-2.260,5)\end{array}$ & $\begin{array}{c}2.180,2 \\
(2.151,7-2.208,7)\end{array}$ & $\begin{array}{c}1.983,3 \\
(1.917,3-2.049,3)\end{array}$ & $\begin{array}{c}1.921,2 \\
(1.878,0-1.964,4)\end{array}$ \\
\hline Potássio (mg) & $\begin{array}{c}2.100,3 \\
(2.052,8-2.147,7)\end{array}$ & $\begin{array}{c}1.906,2 \\
(1.863,0-1.949,4)\end{array}$ & $\begin{array}{c}2.140,5 \\
(2.111,4-2.169,6)\end{array}$ & $\begin{array}{c}1.972,3 \\
(1.948,7-1.996,0)\end{array}$ & $\begin{array}{c}2.109,0 \\
(2.057,3-2.160,8)\end{array}$ & $\begin{array}{c}1.948,3 \\
(1.911,5-1.985,1)\end{array}$ \\
\hline Cobre (mg) & $1,2(1,2-1,3)$ & $1,2(1,2-1,2)$ & $1,2(1,2-1,2)$ & $1,2(1,2-1,2)$ & $1,1(1,1-1,2)$ & $1,2(1,1-1,2)$ \\
\hline Zinco (mg) & $10,2(9,9-10,5)$ & $9,6(9,4-9,8)$ & $10,1(10,0-10,2)$ & $9,5(9,3-9,7)$ & $9,5(9,2-9,8)$ & $8,6(8,4-8,8)$ \\
\hline Vitamina A (mcg) & $\begin{array}{c}400,2 \\
(359,9-440,5)\end{array}$ & $\begin{array}{c}319,6 \\
(289,5-349,8)\end{array}$ & $\begin{array}{c}410,4 \\
(384,6-436,1)\end{array}$ & $\begin{array}{c}342,2 \\
(325,9-358,6)\end{array}$ & $\begin{array}{c}450,8 \\
(429,3-472,4)\end{array}$ & $\begin{array}{c}412 \\
(399,8-424,3)\end{array}$ \\
\hline Tiamina (mg) & $1,0(0,9-1,0)$ & $1,0(1,0-1,0)$ & $0,8(0,8-0,9)$ & $0,9(0,8-0,9)$ & $0,8(0,7-0,8)$ & $0,8(0,8-0,8)$ \\
\hline Riboflavina (mg) & $1,2(1,2-1,3)$ & $1,1(1,0-1,1)$ & $1,2(1,1-1,2)$ & $1,0(0,9-1,0)$ & $1,2(1,1-1,2)$ & $1,0(1,0-1,0)$ \\
\hline Piridoxina (mg) & $0,8(0,7-0,8)$ & $0,7(0,7-0,7)$ & $0,7(0,6-0,7)$ & $0,6(0,6-0,6)$ & $0,6(0,5-0,6)$ & $0,6(0,6-0,6)$ \\
\hline Cobalamina (mcg) & $4,7(4,2-5,3)$ & $3,8(3,5-4,0)$ & $4,6(4,5-4,8)$ & $3,6(3,5-3,7)$ & $4,4(4,3-4,6)$ & $3,3(3,3-3,4)$ \\
\hline Vitamina D $(\mathrm{mcg})^{\mathrm{b}}$ & $2,2(2,1-2,4)$ & $1,8(1,6-1,9)$ & $1,8(1,8-1,9)$ & $1,4(1,4-1,5)$ & $1,8(1,6-2,0)$ & $1,3(1,3-1,3)$ \\
\hline Vitamina $E(m g)^{c}$ & $5,8(5,6-6,0)$ & $5,8(5,4-6,1)$ & $5,5(5,4-5,6)$ & $5,9(5,7-6,1)$ & $5,3(5,3-5,4)$ & $5,5(5,3-5,6)$ \\
\hline Vitamina C (mg) & $\begin{array}{c}128,0 \\
(120,7-135,2)\end{array}$ & $\begin{array}{c}126,0 \\
(117,6-134,4)\end{array}$ & $\begin{array}{c}134,6 \\
(131,7-137,6)\end{array}$ & $\begin{array}{c}120,6 \\
(117,6-123,6)\end{array}$ & $\begin{array}{c}133,5 \\
(122,4-144,7)\end{array}$ & $\begin{array}{c}128,9 \\
(121,2-136,6)\end{array}$ \\
\hline Niacina $(m g)^{d}$ & $13,8(13,2-14,3)$ & $14,6(13,7-15,4)$ & $13,7(13,4-14,0)$ & $14,4(14,0-14,8)$ & $12,6(12,0-13,2)$ & $13,0(12,4-13,5)$ \\
\hline
\end{tabular}

a Equivalente de atividade de retinol (RAE).

${ }^{\mathrm{b}}$ Ergocalciterol (D2) + colecalciterol (D3).

${ }^{c}$ Alfa-tocoferol total.

d Niacina pré-formada. 
As prevalências de inadequação para a maioria dos micronutrientes foram maiores em 2017-2018 que em 2008-2009. As mais importantes diferenças (> 10\%) encontradas na população feminina foram para riboflavina e zinco em todas as faixas de idade e riboflavina entre homens adultos e idosos. De forma contrária, tiamina entre as mulheres idosas e homens de todas as idades, e cobre entre as mulheres adultas e idosas e entre os homens adolescentes e adultos foram os únicos nutrientes que mostraram redução discreta ( $<10$ pontos percentuais) na prevalência de inadequação no período investigado (Tabela 2).

Em 2008-2009, a porcentagem de indivíduos cuja ingestão de sódio deveria ser diminuída para reduzir o risco de doenças crônicas foi, entre os homens, de 75\% (72\%-78\%) em adolescentes, $71 \%$ (70\%-72\%) em adultos, e 52\% (47\%-56\%) em idosos. Entre as mulheres, essas porcentagens foram de $61 \%$ (59\%-63\%), 41\% (39\%-43\%) e $29 \%(28 \%-30 \%)$, respectivamente. Em 2017-2018, os percentuais foram, para o sexo masculino, $78 \%$ (76\%-80\%) em adolescentes, $74 \%(73 \%-75 \%)$ em adultos, e $52 \%(50 \%-54 \%)$ em idosos. Entre as mulheres, $59 \%(56 \%-61 \%), 39 \%(36 \%-41 \%)$ e $25 \%(24 \%-27 \%)$.

Tanto em 2008-2009 (Tabelas 3 e 4) quanto em 2017-2018 (Tabelas 5 e 6), houve redução das prevalências de inadequação dos micronutrientes com o aumento da renda média per capita.

Tabela 2. Prevalências de inadequação (IC95\%) da ingestão de vitaminas e minerais entre adolescentes, adultos e idosos nos Inquéritos Nacionais de Alimentação 2008-2009 e 2017-2018.

\begin{tabular}{|c|c|c|c|c|c|c|}
\hline \multirow{2}{*}{ Nutrientes } & \multicolumn{2}{|c|}{ Adolescentes } & \multicolumn{2}{|c|}{ Adultos } & \multicolumn{2}{|c|}{ Idosos } \\
\hline & 2008-2009 & 2017-2018 & 2008-2009 & 2017-2018 & 2008-2009 & 2017-2018 \\
\hline \multicolumn{7}{|l|}{ Homens } \\
\hline Cálcio (mg) & $97,4(96,9-97,9)$ & $98,1(97,9-98,4)$ & $89,1(88,0-90,2)$ & $91,0(90,1-91,8)$ & $92,4(91,0-93,8)$ & $94,4(93,6-95,1)$ \\
\hline Magnésio (mg) & $50,2(48,5-52,0)$ & $54,2(51,2-57,2)$ & $66,1(65,2-67,1)$ & $69,2(67,9-70,5)$ & $77,0(74,4-79,5)$ & $80,5(78,7-82,3)$ \\
\hline Fósforo (mg) & $50,3(47,8-52,8)$ & $51,8(49,9-53,7)$ & $2,8(2,3-3,4)$ & $2,1(1,8-2,5)$ & $6,5(5,7-7,4)$ & $6,9(6,4-7,5)$ \\
\hline Cobre (mg) & $3,1(2,7-3,6)$ & $2,1(1,7-2,4)$ & $3,7(3,2-4,1)$ & $2,4(2,1-2,7)$ & $6,4(4,7-8,1)$ & $4,5(4,0-5,0)$ \\
\hline Zinco (mg) & $16,5(13,9-19,0)$ & $17,5(14,7-20,3)$ & $22,4(21,1-23,6)$ & $23,5(22,4-24,7)$ & $33,5(30,8-36,1)$ & $39,0(37,7-40,2)$ \\
\hline Vitamina A $(\mathrm{mcg})^{\mathrm{a}}$ & $78,8(75,4-82,3)$ & $83,5(81,1-85,9)$ & $84,7(82,9-86,4)$ & $89,3(88,4-90,2)$ & $84,7(82,9-86,6)$ & $87,5(86,1-89,0)$ \\
\hline Tiamina (mg) & $41,1(38,1-44,1)$ & $32,0(29,7-34,4)$ & $59,8(56,8-62,8)$ & $51,8(50,9-52,7)$ & $70,0(68,1-72,0)$ & $63,6(61,8-65,4)$ \\
\hline Riboflavina (mg) & $31,4(26,7-36,0)$ & $36,6(34,3-38,9)$ & $45,9(43,9-47,8)$ & $55,0(54,1-55,9)$ & $47,9(45,2-50,5)$ & $57,9(56,9-58,8)$ \\
\hline Piridoxina (mg) & $70,2(68,6-71,8)$ & $68,8(65,7-72,0)$ & $81,5(77,9-85,0)$ & $82,3(80,5-84,0)$ & $95,9(94,5-97,2)$ & $95,0(94,3-95,7)$ \\
\hline Cobalamina (mcg) & $6,2(4,3-8,1)$ & $7,7(6,4-9,1)$ & $5,5(4,3-6,6)$ & $7,2(6,2-8,1)$ & $7,9(6,2-9,6)$ & $13,3(11,9-14,7)$ \\
\hline Vitamina D $(\mathrm{mcg})^{\mathrm{b}}$ & $99,4(99,2-99,6)$ & $99,8(99,8-99,9)$ & $99,6(99,4-99,8)$ & $99,9(99,9-100)$ & $99,7(99,6-99,9)$ & $100(99,9-100,0)$ \\
\hline Vitamina $E(m g)^{c}$ & $90,6(89,5-91,7)$ & $89,1(87,4-90,8)$ & $93,6(93,0-94,1)$ & $91,6(90,4-92,8)$ & $96,1(95,3-96,8)$ & $95,3(94,2-96,3)$ \\
\hline Vitamina C (mg) & $33,2(31,7-34,6)$ & $34,5(30,0-38,9)$ & $43,7(42,8-44,6)$ & $47,5(45,8-49,2)$ & $45,3(42,8-47,8)$ & $48,7(46,2-51,3)$ \\
\hline \multicolumn{7}{|l|}{ Mulheres } \\
\hline Cálcio (mg) & $98,0(97,6-98,3)$ & $99,0(98,5-99,4)$ & $93,7(93,1-94,3)$ & $96,1(95,8-96,5)$ & $97,5(96,9-98,1)$ & $98,5(98,3-98,6)$ \\
\hline Magnésio (mg) & $57,8(54,2-61,4)$ & $64,7(62,5-66,9)$ & $64,1(63,3-64,9)$ & $68,8(66,9-70,7)$ & $69,1(68,0-70,1)$ & $73,0(71,8-74,1)$ \\
\hline Fósforo (mg) & $64,0(62,2-65,7)$ & $71,1(66,8-75,3)$ & $11,5(10,8-12,2)$ & $12,8(12,0-13,6)$ & $15,0(14,1-15,9)$ & $18,7(17,2-20,1)$ \\
\hline Cobre (mg) & $7,3(6,3-8,3)$ & $6,6(5,9-7,2)$ & $13,1(12,0-14,3)$ & $10,6(9,9-11,3)$ & $15,0(13,8-16,3)$ & $11,9(10,3-13,5)$ \\
\hline Zinco (mg) & $21,1(18,8-23,4)$ & $25,6(23,7-27,5)$ & $18,1(17,1-19,1)$ & $22,2(20,7-23,8)$ & $23,0(20,2-25,7)$ & $31,4(29,2-33,7)$ \\
\hline Vitamina A $(\mathrm{mcg})^{\mathrm{a}}$ & $69,2(64,9-73,4)$ & $78,9(75,3-82,5)$ & $72,4(69,7-75,1)$ & $80,1(78,5-81,6)$ & $68,0(65,7-70,3)$ & $72,3(71,1-73,6)$ \\
\hline Tiamina (mg) & $42,5(40,2-44,8)$ & $40,8(39,3-42,3)$ & $63,5(62,4-64,6)$ & $61,8(60,6-63,1)$ & $70,2(67,3-73,1)$ & $66,5(65,8-67,2)$ \\
\hline Riboflavina (mg) & $27,1(22,2-31,9)$ & $40,2(34,4-46,1)$ & $36,6(34,5-38,6)$ & $51,4(50,0-52,8)$ & $36,6(32,6-40,6)$ & $46,2(44,7-47,8)$ \\
\hline Piridoxina (mg) & $71,2(68,6-73,8)$ & $75,8(72,6-78,9)$ & $89,5(87,8-91,3)$ & $91,8(91,1-92,5)$ & $96,4(95,1-97,8)$ & $96,7(96,2-97,2)$ \\
\hline Cobalamina (mcg) & $7,6(4,4-10,8)$ & $11,4(7,6-15,3)$ & $10,9(9,2-12,5)$ & $17,1(15,5-18,8)$ & $12,6(11,1-14,0)$ & $21,3(18,2-24,4)$ \\
\hline Vitamina D $(\mathrm{mcg})^{\mathrm{b}}$ & $99,5(99,3-99,6)$ & $99,9(99,8-100,0)$ & $99,8(99,7-99,9)$ & $100,0(99,9-100)$ & $99,8(99,6-100,0)$ & $100,0(100,0-100,0)$ \\
\hline Vitamina $E(m g)^{c}$ & $94,1(93,2-94,9)$ & $93,9(92,1-95,6)$ & $98,1(97,9-98,4)$ & $97,0(96,1-97,8)$ & $98,4(98,2-98,6)$ & $98,0(97,4-98,6)$ \\
\hline Vitamina C (mg) & $29(26,3-31,7)$ & $29,4(25,7-33,1)$ & $34,4(33,5-35,2)$ & $38,5(37,1-39,9)$ & $34,7(31,6-37,9)$ & $35,7(32,9-38,6)$ \\
\hline
\end{tabular}

a Equivalente de atividade de retinol (RAE).

${ }^{\mathrm{b}}$ Ergocalciterol (D2) + colecalciterol (D3).

c Alfa-tocoferol total. 
Tabela 3. Prevalências de inadequação (IC95\%) da ingestão de vitaminas e minerais segundo renda familiar média per capita entre homens no Inquérito Nacional de Alimentação (INA/POF) 2008-2009.

\begin{tabular}{|c|c|c|c|c|c|}
\hline \multirow{3}{*}{ Nutrientes } & \multirow{3}{*}{ Faixa etária } & \multicolumn{4}{|c|}{ Renda per capita } \\
\hline & & $<0,5 \mathrm{SM}$ & 0,5-1 SM & $1-2$ SM & $>2 \mathrm{SM}$ \\
\hline & & $\%$ (IC95\%) & $\%($ IC95\%) & $\%($ IC95\%) & $\%($ IC95\%) \\
\hline \multicolumn{6}{|l|}{ Cálcio (mg) } \\
\hline & Adolescentes & $99,3(98,9-99,6)$ & $98,5(98,3-98,8)$ & $97,2(96,4-98,0)$ & $92,4(90,9-94,0)$ \\
\hline & Adultos & $96,7(96,1-97,3)$ & $94,2(93,8-94,7)$ & $90,7(89,6-91,8)$ & $80,7(78,5-83,0)$ \\
\hline & Idosos & $98,4(97,9-98,9)$ & $96,8(96,1-97,4)$ & $95,1(94,5-95,7)$ & $87,4(85,1-89,7)$ \\
\hline \multicolumn{6}{|c|}{ Magnésio (mg) } \\
\hline & Adolescentes & $50,0(45,2-54,8)$ & $51,0(48,1-53,9)$ & $48,1(45,6-50,5)$ & $53,3(50,3-56,2)$ \\
\hline & Adultos & $71,1(69,1-73,1)$ & $65,3(62,8-67,7)$ & $63,6(62,5-64,7)$ & $66,6(64,4-68,9)$ \\
\hline & Idosos & $81,4(77,0-85,9)$ & $76,4(72,4-80,4)$ & $76,1(72,3-79,9)$ & $77,6(76,1-79,0)$ \\
\hline \multicolumn{6}{|c|}{ Fósforo (mg) } \\
\hline & Adolescentes & $62,2(60,0-64,3)$ & $50,3(48,1-52,6)$ & $45,4(42,6-48,1)$ & $36,1(33,5-38,7)$ \\
\hline & Adultos & $5,6(4,9-6,3)$ & $3,3(2,4-4,2)$ & $2,5(2,1-3,0)$ & $1,5(1,1-1,9)$ \\
\hline & Idosos & $13,4(10,8-16,1)$ & $8,2(6,7-9,7)$ & $7,2(5,6-8,7)$ & $4,1(3,6-4,6)$ \\
\hline \multicolumn{6}{|l|}{ Cobre (mg) } \\
\hline & Adolescentes & $3,6(2,7-4,4)$ & $2,9(2,5-3,4)$ & $2,7(2,1-3,3)$ & $3,5(3,0-3,9)$ \\
\hline & Adultos & $4,6(3,7-5,5)$ & $3,3(2,9-3,8)$ & $3,3(2,7-4,0)$ & $3,7(3,1-4,4)$ \\
\hline & Idosos & $8,4(5,5-11,4)$ & $6,0(4,2-7,7)$ & $6,4(4,0-8,8)$ & $6,3(5,0-7,5)$ \\
\hline \multicolumn{6}{|l|}{ Zinco (mg) } \\
\hline & Adolescentes & $21,4(16,2-26,6)$ & $15,3(13,9-16,7)$ & $14,0(12,1-16,0)$ & $14,0(12,6-15,4)$ \\
\hline & Adultos & $30,3(28,2-32,4)$ & $22,1(19,7-24,5)$ & $20,8(19,7-22,0)$ & $20,1(18,2-22,0)$ \\
\hline & Idosos & $44,4(40,4-48,4)$ & $34,0(31,3-36,7)$ & $34,3(31,6-37,0)$ & $31,0(26,6-35,4)$ \\
\hline \multicolumn{6}{|c|}{ Vitamina A (mcg) } \\
\hline & Adolescentes & $87,0(82,1-91,9)$ & $81,6(79,0-84,3)$ & $76,0(73,8-78,2)$ & $61,6(58,7-64,6)$ \\
\hline & Adultos & $95,2(93,4-96,9)$ & $90,2(88,7-91,7)$ & $86,6(84,7-88,4)$ & $73,9(70,9-76,9)$ \\
\hline & Idosos & $96,1(93,9-98,2)$ & $92,3(90,6-93,9)$ & $88,8(87,5-90,2)$ & $77,1(74,3-79,9)$ \\
\hline \multicolumn{6}{|c|}{ Tiamina (mg) } \\
\hline & Adolescentes & $52,8(45,3-60,3)$ & $41,6(38,6-44,6)$ & $34,5(32,6-36,5)$ & $28,5(25,9-31,1)$ \\
\hline & Adultos & $78,0(74,9-81,0)$ & $64,4(60,9-67,8)$ & $57,9(55,8-60,0)$ & $49,6(43,9-55,2)$ \\
\hline & Idosos & $87,8(84,4-91,3)$ & $77,2(74,4-80,1)$ & $72,0(68,0-76,0)$ & $62,8(59,9-65,8)$ \\
\hline \multicolumn{6}{|c|}{ Riboflavina (mg) } \\
\hline & Adolescentes & $42,8(35,6-50,0)$ & $30,6(26,3-34,9)$ & $25,5(22,8-28,3)$ & $20,1(17,7-22,6)$ \\
\hline & Adultos & $65,7(61,1-70,2)$ & $49,3(46,9-51,7)$ & $43,8(42,3-45,2)$ & $35,7(32,9-38,6)$ \\
\hline & Idosos & $71,3(68,1-74,5)$ & $54,9(50,9-58,9)$ & $49,9(47,7-52,2)$ & $39,8(36,1-43,6)$ \\
\hline \multicolumn{6}{|c|}{ Piridoxina (mg) } \\
\hline & Adolescentes & $81,5(78,1-84,8)$ & $71,3(69,1-73,4)$ & $64,6(62,3-67,0)$ & $55,2(52,7-57,6)$ \\
\hline & Adultos & $92,9(91,1-94,6)$ & $85,1(82,1-88,1)$ & $81,4(77,9-84,8)$ & $73,6(68,2-79,0)$ \\
\hline & Idosos & $99,5(99,2-99,8)$ & $97,8(97,2-98,5)$ & $97,0(96,3-97,8)$ & $94,0(92,5-95,6)$ \\
\hline \multicolumn{6}{|c|}{ Cobalamina (mcg) } \\
\hline & Adolescentes & $7,6(5,2-9,9)$ & $6,6(4,7-8,5)$ & $5,4(3,9-7,0)$ & $3,8(2,6-4,9)$ \\
\hline & Adultos & $8,1(6-10,3)$ & $6,4(5,1-7,8)$ & $5,5(4,4-6,7)$ & $3,5(2,5-4,6)$ \\
\hline & Idosos & $12,8(10,9-14,6)$ & $9,8(7,7-12)$ & $9,2(7,7-10,7)$ & $5,3(3,5-7,1)$ \\
\hline \multicolumn{6}{|l|}{$\begin{array}{l}\text { Vitamina D } \\
\left(\text { (mcg }^{b}\right.\end{array}$} \\
\hline & Adolescentes & $99,5(99,3-99,7)$ & $99,5(99,4-99,6)$ & $99,3(99,1-99,6)$ & $98,9(98,5-99,2)$ \\
\hline & Adultos & $99,7(99,6-99,9)$ & $99,7(99,5-99,8)$ & $99,7(99,5-99,8)$ & $99,3(99,0-99,7)$ \\
\hline & Idosos & $99,9(99,7-100)$ & $99,8(99,7-100)$ & $99,7(99,6-99,9)$ & $99,6(99,3-99,9)$ \\
\hline \multicolumn{6}{|c|}{ Vitamina $\mathrm{E}(\mathrm{mg})^{\mathrm{c}}$} \\
\hline & Adolescentes & $91,5(89,2-93,7)$ & $90,3(89,2-91,4)$ & $89,8(88,6-91,1)$ & $90,1(89,3-90,9)$ \\
\hline & Adultos & $95,3(94,7-95,8)$ & $93,3(92,0-94,6)$ & $93,4(93,2-93,7)$ & $93,1(92,1-94,1)$ \\
\hline & Idosos & $97,3(96,5-98,2)$ & $96,0(95,3-96,7)$ & $96,4(95,9-96,8)$ & $95,7(94,4-96,9)$ \\
\hline \multicolumn{6}{|c|}{ Vitamina C (mg) } \\
\hline & Adolescentes & $43,8(40,6-46,9)$ & $35,2(33,4-36,9)$ & $27,5(25,9-29,1)$ & $17,8(16,1-19,5)$ \\
\hline & Adultos & $64,2(61,3-67,2)$ & $51,5(49,3-53,6)$ & $43,1(41,2-45,1)$ & $29,3(27,4-31,2)$ \\
\hline & Idosos & $69,8(65,6-74,0)$ & $57,3(54,6-59,9)$ & $48,7(46,3-51,2)$ & $33,2(30,1-36,3)$ \\
\hline
\end{tabular}

${ }^{a}$ Equivalente de atividade de retinol (RAE).

${ }^{\mathrm{b}}$ Ergocalciterol (D2) + colecalciterol (D3).

c Alfa-tocoferol total. 
Tabela 4. Prevalências de inadequação (IC95\%) da ingestão de vitaminas e minerais segundo renda média familiar per capita entre mulheres no Inquérito Nacional de Alimentação (INA/POF) 2008-2009.

\begin{tabular}{|c|c|c|c|c|c|}
\hline \multirow{3}{*}{ Nutrientes } & \multirow{3}{*}{ Faixa etária } & \multicolumn{4}{|c|}{ Renda per capita } \\
\hline & & $<0,5 \mathrm{SM}$ & 0,5-1 SM & 1-2 SM & $>2 \mathrm{SM}$ \\
\hline & & $\%($ (IC95\%) & $\%($ IC95\%) & \% (IC95\%) & $\%$ (IC95\%) \\
\hline \multicolumn{6}{|l|}{ Cálcio (mg) } \\
\hline & Adolescentes & $99,3(99,1-99,5)$ & $98,9(98,7-99,1)$ & $97,9(97,5-98,2)$ & $94,2(93,3-95,1)$ \\
\hline & Adultos & $98,2(97,9-98,6)$ & $96,9(96,6-97,2)$ & $94,7(94,1-95,2)$ & $87,8(86,3-89,4)$ \\
\hline & Idosos & $99,6(99,6-99,7)$ & $99,3(99,2-99,4)$ & $98,6(98,3-99,0)$ & $96,0(94,8-97,2)$ \\
\hline \multicolumn{6}{|c|}{ Magnésio (mg) } \\
\hline & Adolescentes & $58,9(55,8-62,0)$ & $56,4(52,4-60,4)$ & $56,6(52,0-61,3)$ & $60,8(52,9-68,7)$ \\
\hline & Adultos & $69,5(67,4-71,5)$ & $63,3(61,3-65,3)$ & $61,5(60,3-62,7)$ & $64,5(62,5-66,5)$ \\
\hline & Idosos & $73,3(70,8-75,9)$ & $69,2(66,9-71,5)$ & $67,1(65,2-69,1)$ & $69,9(68,3-71,6)$ \\
\hline \multicolumn{6}{|c|}{ Fósforo (mg) } \\
\hline & Adolescentes & $73,2(70,6-75,8)$ & $65,1(63,5-66,8)$ & $59,5(56,7-62,3)$ & $51,8(49,8-53,8)$ \\
\hline & Adultos & $19,1(17,5-20,8)$ & $13,3(12,0-14,6)$ & $10,4(9,4-11,3)$ & $7,3(6,9-7,7)$ \\
\hline & Idosos & $26,5(25,0-28,0)$ & $19,7(17,6-21,8)$ & $15,7(14,1-17,4)$ & $11,1(10,1-12)$ \\
\hline \multicolumn{6}{|l|}{ Cobre (mg) } \\
\hline & Adolescentes & $8,1(6,4-9,8)$ & $6,7(5,9-7,5)$ & $6,8(5,7-7,9)$ & $7,6(6,4-8,8)$ \\
\hline & Adultos & $15,7(13,4-17,9)$ & $12,6(11,8-13,4)$ & $12,1(10,1-14,1)$ & $13,2(12,5-14,0)$ \\
\hline & Idosos & $17,7(14,6-20,7)$ & $14,8(13,1-16,5)$ & $14,4(12,4-16,4)$ & $15,3(13,8-16,9)$ \\
\hline \multicolumn{6}{|l|}{ Zinco (mg) } \\
\hline & Adolescentes & $26,6(22,1-31,2)$ & $19,9(18,9-20,8)$ & $18,4(15,7-21,0)$ & $17,7(16,1-19,3)$ \\
\hline & Adultos & $25,1(22,3-27,9)$ & $18,0(16,5-19,4)$ & $16,6(15,4-17,7)$ & $16,2(15,4-17,0)$ \\
\hline & Idosos & $31,9(26,3-37,5)$ & $24,4(21,4-27,4)$ & $22,5(18,9-26,0)$ & $21,6(19,6-23,7)$ \\
\hline \multicolumn{6}{|c|}{ Vitamina A (mcg) $)^{a}$} \\
\hline & Adolescentes & $81,4(75,9-86,9)$ & $71,6(68,6-74,7)$ & $65,6(63,4-67,7)$ & $48,0(44,7-51,3)$ \\
\hline & Adultos & $88,1(84,8-91,4)$ & $79,6(77,3-81,9)$ & $74,3(71,8-76,8)$ & $57,1(52,8-61,3)$ \\
\hline & Idosos & $88,2(84,4-91,9)$ & $79,8(77,7-81,8)$ & $73,6(71,4-75,9)$ & $56,7(53,9-59,5)$ \\
\hline \multicolumn{6}{|c|}{ Tiamina (mg) } \\
\hline & Adolescentes & $55,5(50,0-61,1)$ & $41,9(39,8-44,0)$ & $36,1(34,0-38,3)$ & $29,4(25,9-32,9)$ \\
\hline & Adultos & $80,5(77,4-83,7)$ & $67,6(65,3-70,0)$ & $61,4(60,1-62,8)$ & $53,3(50,5-56,2)$ \\
\hline & Idosos & $88,5(85,8-91,2)$ & $78,2(74,5-81,9)$ & $71,7(69,4-74,0)$ & $64,4(59,4-69,3)$ \\
\hline \multicolumn{6}{|c|}{ Riboflavina (mg) } \\
\hline & Adolescentes & $39,6(32,8-46,4)$ & $25,6(20,8-30,5)$ & $21,3(18,1-24,6)$ & $15,5(11,5-19,6)$ \\
\hline & Adultos & $56,5(51,9-61,0)$ & $39,6(36,4-42,8)$ & $34,0(32,9-35,1)$ & $26,6(24,2-28,9)$ \\
\hline & Idosos & $60,5(53,3-67,6)$ & $44,0(40,3-47,6)$ & $37,4(33,9-40,9)$ & $29,7(25,7-33,7)$ \\
\hline \multicolumn{6}{|c|}{ Piridoxina (mg) } \\
\hline & Adolescentes & $82,9(80,4-85,5)$ & $71,5(67,8-75,2)$ & $66,0(61,8-70,2)$ & $56,0(50,1-61,9)$ \\
\hline & Adultos & $96,7(95,8-97,6)$ & $92,1(90,4-93,7)$ & $89,5(87,6-91,4)$ & $84,0(81,4-86,6)$ \\
\hline & Idosos & $99,5(99,4-99,7)$ & $98,3(97,6-99,0)$ & $97,4(96,5-98,3)$ & $95,3(93,4-97,1)$ \\
\hline \multicolumn{6}{|c|}{ Cobalamina (mcg) } \\
\hline & Adolescentes & $9,2(5,7-12,7)$ & $8,1(4,3-11,9)$ & $6,9(4,7-9,2)$ & $4,5(2,4-6,6)$ \\
\hline & Adultos & $15,2(12,8-17,7)$ & $12,7(10,4-15,1)$ & $10,7(9,2-12,2)$ & $7,5(6,1-8,8)$ \\
\hline & Idosos & $18,9(16,0-21,9)$ & $16,4(14,2-18,6)$ & $13,6(12,4-14,9)$ & $9,4(7,4-11,3)$ \\
\hline \multicolumn{6}{|l|}{$\begin{array}{l}\text { Vitamina D } \\
(\mathrm{mcg})^{\mathrm{b}}\end{array}$} \\
\hline & Adolescentes & $99,6(99,4-99,7)$ & $99,6(99,5-99,7)$ & $99,5(99,3-99,7)$ & $99,1(98,8-99,3)$ \\
\hline & Adultos & $99,9(99,8-100)$ & $99,8(99,8-99,9)$ & $99,8(99,8-99,9)$ & $99,6(99,5-99,8)$ \\
\hline & Idosos & $99,9(99,8-100)$ & $99,9(99,8-100)$ & $99,8(99,8-99,9)$ & $99,7(99,3-100,1)$ \\
\hline \multicolumn{6}{|c|}{ Vitamina $E(m g)^{c}$} \\
\hline & Adolescentes & $94,5(93,5-95,6)$ & $93,7(92,1-95,3)$ & $94,0(93,0-94,9)$ & $93,9(92,1-95,7)$ \\
\hline & Adultos & $98,6(98,5-98,8)$ & $98,1(97,6-98,6)$ & $98,1(97,9-98,2)$ & $97,9(97,6-98,2)$ \\
\hline & Idosos & $99,1(98,8-99,4)$ & $98,5(98,0-99,0)$ & $98,4(98,3-98,6)$ & $98,3(98,1-98,6)$ \\
\hline \multicolumn{6}{|c|}{ Vitamina C (mg) } \\
\hline & Adolescentes & $41,2(36,9-45,5)$ & $30,1(26,5-33,6)$ & $23,3(19,1-27,5)$ & $13,5(10,3-16,7)$ \\
\hline & Adultos & $54,1(51,3-56,9)$ & $41,1(39,1-43,0)$ & $32,9(31,4-34,5)$ & $20,8(18,6-23,0)$ \\
\hline & Idosos & $58,6(53,8-63,4)$ & $46,3(42,6-49,9)$ & $37,4(32,9-41,8)$ & $24,3(21,4-27,2)$ \\
\hline
\end{tabular}

${ }^{a}$ Equivalente de atividade de retinol (RAE).

${ }^{\mathrm{b}}$ Ergocalciterol (D2) + colecalciterol (D3).

${ }^{c}$ Alfa-tocoferol total. 
Tabela 5. Prevalências de inadequação (IC95\%) da ingestão de vitaminas e minerais segundo renda familiar per capita entre homens no İnquérito Nacional de Alimentação 2017-2018.

\begin{tabular}{|c|c|c|c|c|c|}
\hline \multirow{2}{*}{ Nutrientes } & \multirow{2}{*}{ Faixa etária } & \multicolumn{4}{|c|}{ Renda per capita } \\
\hline & & $<0.5 \mathrm{SM}$ & 0.5-1 SM & 1-2 SM & $>2 \mathrm{SM}$ \\
\hline \multicolumn{6}{|l|}{ Cálcio (mg) } \\
\hline & Adolescentes & $99,6(99,5-99,8)$ & 99,0 (98,9-99,2) & $97,8(97,3-98,3)$ & $93,1(92,1-94,2)$ \\
\hline & Adultos & $98,1(97,7-98,5)$ & $95,8(95,2-96,4)$ & $91,7(90,4-93,0)$ & $81,9(79,8-83,9)$ \\
\hline & Idosos & $99,2(99,0-99,5)$ & $98,2(97,8-98,7)$ & $96,6(95,5-97,6)$ & $90,2(89,1-91,3)$ \\
\hline \multicolumn{6}{|c|}{ Magnésio (mg) } \\
\hline & Adolescentes & $57,4(54,7-60,1)$ & $51,8(48,6-54,9)$ & $53,9(47,7-60,0)$ & $53,1(45,4-60,9)$ \\
\hline & Adultos & $73,9(70,1-77,7)$ & $67,4(65,6-69,3)$ & $68,4(64,7-72,0)$ & $69,1(66,8-71,4)$ \\
\hline & Idosos & $83,7(81,2-86,3)$ & $78,9(77,7-80,1)$ & $80,9(77,9-83,8)$ & $80,5(78,7-82,2)$ \\
\hline \multicolumn{6}{|c|}{ Fósforo (mg) } \\
\hline & Adolescentes & $63,1(59,7-66,4)$ & $51,8(48,6-55,0)$ & $45,5(41-50,0)$ & $38,3(35,0-41,5)$ \\
\hline & Adultos & $4,5(2,9-6,0)$ & $2,3(1,9-2,7)$ & $1,8(1,6-2,0)$ & $1,1(0,8-1,3)$ \\
\hline & Idosos & $14(11,9-16,1)$ & $9,0(8,3-9,7)$ & $7,2(6,4-8,1)$ & $4,5(4-5,1)$ \\
\hline \multicolumn{6}{|l|}{ Cobre (mg) } \\
\hline & Adolescentes & $2,2(1,7-2,6)$ & $1,8(1,4-2,1)$ & $2,3(1,6-3,1)$ & $2,2(1,5-3,0)$ \\
\hline & Adultos & $2,4(1,6-3,2)$ & $2,0(1,6-2,3)$ & $2,5(2,1-3,0)$ & $2,7(2,3-3,1)$ \\
\hline & Idosos & $4,1(2,7-5,5)$ & $3,6(3,0-4,1)$ & $4,7(3,9-5,6)$ & $4,7(4,3-5,1)$ \\
\hline \multicolumn{6}{|l|}{ Zinco (mg) } \\
\hline & Adolescentes & $20,6(17,0-24,3)$ & $17,6(14,3-20,9)$ & $15,5(14,0-16,9)$ & $14,1(11,7-16,5)$ \\
\hline & Adultos & $28,7(26,4-31,1)$ & $24,6(22,8-26,5)$ & $22,3(20,3-24,3)$ & $21,0(18,5-23,5)$ \\
\hline & Idosos & $45,3(41,9-48,8)$ & $41,1(38,1-44,1)$ & $39,4(37,6-41,2)$ & $36,5(35,3-37,7)$ \\
\hline \multicolumn{6}{|c|}{ Vitamina A (mcg) $)^{a}$} \\
\hline & Adolescentes & $92,7(90,2-95,3)$ & $85,8(82,3-89,3)$ & $78,6(73,7-83,4)$ & $64,6(60,2-69,0)$ \\
\hline & Adultos & $97,7(97,1-98,3)$ & $94,3(93,6-95,0)$ & $89,3(87,8-90,9)$ & $79,7(78,1-81,3)$ \\
\hline & Idosos & $98,1(97,7-98,6)$ & $94,7(93,7-95,8)$ & $90,2(88,6-91,8)$ & $80,7(78,8-82,6)$ \\
\hline \multicolumn{6}{|c|}{ Tiamina (mg) } \\
\hline & Adolescentes & $42,6(37,7-47,6)$ & $30,1(27,6-32,6)$ & $26,5(24,6-28,5)$ & $23,2(21,1-25,3)$ \\
\hline & Adultos & $67,4(63,7-71,2)$ & $53,3(51,9-54,6)$ & $48,8(45,8-51,8)$ & $45,2(43,2-47,2)$ \\
\hline & Idosos & $79,7(77,3-82,1)$ & $67,6(65,3-69,9)$ & $63,9(59,7-68)$ & $59,5(58,2-60,7)$ \\
\hline \multicolumn{6}{|c|}{ Riboflavina (mg) } \\
\hline & Adolescentes & $46,9(44,2-49,6)$ & $35,9(32,0-39,8)$ & $31,3(27,0-35,7)$ & $25,1(22,7-27,5)$ \\
\hline & Adultos & $70,4(68,4-72,4)$ & $58,3(56,4-60,3)$ & $52,8(49,8-55,8)$ & $46,1(43,6-48,6)$ \\
\hline & Idosos & $75,7(73,6-77,7)$ & $64,1(62,6-65,5)$ & $58,8(56,1-61,5)$ & $51,7(48,5-54,9)$ \\
\hline \multicolumn{6}{|c|}{ Piridoxina (mg) } \\
\hline & Adolescentes & $77,5(75,6-79,4)$ & $68,6(63,4-73,9)$ & $64,7(58,8-70,5)$ & $57,0(53,1-60,8)$ \\
\hline & Adultos & $90,2(88,1-92,3)$ & $84,2(82,0-86,4)$ & $81,5(79,9-83,0)$ & $77,0(74,0-79,9)$ \\
\hline & Idosos & $98,5(98,2-98,7)$ & $96,7(95,7-97,6)$ & $95,7(94,8-96,6)$ & $93,3(92,4-94,1)$ \\
\hline \multicolumn{6}{|c|}{ Cobalamina (mcg) } \\
\hline & Adolescentes & $9,2(7,3-11,1)$ & $9,0(7,6-10,5)$ & $6,3(5,3-7,3)$ & $2,6(1,7-3,5)$ \\
\hline & Adultos & $10,4(9,2-11,7)$ & $10,0(8,5-11,5)$ & $7,0(5,1-8,8)$ & $3,4(3,0-3,8)$ \\
\hline & Idosos & $19,8(17,8-21,8)$ & $19,8(17,8-21,8)$ & $15,1(12,2-18)$ & $7,7(6,9-8,4)$ \\
\hline \multicolumn{6}{|l|}{$\begin{array}{l}\text { Vitamina D } \\
(\mathrm{mcg})^{\mathrm{b}}\end{array}$} \\
\hline & Adolescentes & $99,9(99,9-100)$ & 99,9 (99,9-99,9) & $99,8(99,7-99,8)$ & 99,3 (99-99,5) \\
\hline & Adultos & $100(100-100)$ & $100(100-100)$ & $99,9(99,9-100)$ & $99,8(99,7-99,9)$ \\
\hline & Idosos & $100(100-100)$ & $100(100-100)$ & $100(100-100)$ & $99,9(99,9-100)$ \\
\hline \multicolumn{6}{|c|}{ Vitamina $E(m g)^{c}$} \\
\hline & Adolescentes & $91,4(89,7-93,1)$ & $89,4(87,7-91)$ & $88,3(85-91,6)$ & $84,6(81,0-88,3)$ \\
\hline & Adultos & $94,4(92,7-96,2)$ & $92,7(91,7-93,8)$ & $91,4(89,5-93,3)$ & $89,2(88,0-90,5)$ \\
\hline & Idosos & $97,2(96,0-98,5)$ & $96,2(95,3-97,2)$ & $95,8(94,6-97)$ & $94,0(92,9-95,1)$ \\
\hline \multicolumn{6}{|c|}{ Vitamina C (mg) } \\
\hline & Adolescentes & $47,7(41,0-54,5)$ & $34,1(31,4-36,7)$ & $27,6(24,9-30,2)$ & $17,8(16,0-19,7)$ \\
\hline & Adultos & $68,0(63,9-72,2)$ & $53,2(51,7-54,7)$ & $45,5(44,3-46,6)$ & $33,3(32,1-34,5)$ \\
\hline & Idosos & $72,8(70,0-75,5)$ & $58,5(55,0-62,1)$ & $51,3(48,7-53,8)$ & $38,0(34,6-41,3)$ \\
\hline
\end{tabular}

SM: salário-mínimo.

a Equivalente de atividade de retinol (RAE).

${ }^{\mathrm{b}}$ Ergocalciterol (D2) + colecalciterol (D3).

c Alfa-tocoferol total. 
Tabela 6. Prevalências de inadequação (IC95\%) da ingestão de vitaminas e minerais segundo renda familiar per capita entre mulheres no Inquérito Nacional de Alimentação 2017-2018.

\begin{tabular}{|c|c|c|c|c|c|}
\hline \multirow{2}{*}{ Nutrientes } & \multirow{2}{*}{ Faixa etária } & \multicolumn{4}{|c|}{ Renda per capita } \\
\hline & & $<0.5 \mathrm{SM}$ & 0.5-1 SM & 1-2 SM & $>2 \mathrm{SM}$ \\
\hline \multicolumn{6}{|l|}{ Cálcio (mg) } \\
\hline & Adolescentes & $99,8(99,7-99,9)$ & $99,5(99,2-99,8)$ & $98,8(98,0-99,6)$ & $96,1(95,0-97,1)$ \\
\hline & Adultos & $99,3(99,2-99,4)$ & $98,4(97,9-98,9)$ & $96,5(95,3-97,8)$ & $91,3(90,6-92,0)$ \\
\hline & Idosos & $99,9(99,9-100)$ & $99,7(99,7-99,8)$ & $99,2(99,0-99,5)$ & $97,3(96,9-97,6)$ \\
\hline \multicolumn{6}{|c|}{ Magnésio (mg) } \\
\hline & Adolescentes & $65,9(61,7-70,2)$ & $63,0(60,7-65,3)$ & $65,5(58,9-72,1)$ & $63,9(58,9-68,8)$ \\
\hline & Adultos & $73,2(69,9-76,5)$ & $67,2(65,6-68,7)$ & $67,9(63,7-72,1)$ & $68,6(65,4-71,8)$ \\
\hline & Idosos & $77,3(71,6-83,0)$ & $72,1(68,4-75,8)$ & $73,0(71,3-74,7)$ & $72,8(72,3-73,4)$ \\
\hline \multicolumn{6}{|c|}{ Fósforo (mg) } \\
\hline & Adolescentes & $80,3(77,4-83,2)$ & $70,9(66,4-75,3)$ & $66,2(59,4-73,0)$ & $57,7(51,5-64,0)$ \\
\hline & Adultos & $20,9(18,3-23,5)$ & $13,9(12,8-15,0)$ & $11,3(9,7-12,9)$ & $8,2(7,2-9,2)$ \\
\hline & Idosos & $32,5(26,4-38,7)$ & $23,1(21,8-24,4)$ & $19,0(17,9-20,2)$ & $13,8(12,5-15,1)$ \\
\hline \multicolumn{6}{|l|}{ Cobre (mg) } \\
\hline & Adolescentes & $6,2(5,1-7,3)$ & $5,9(5-6,7)$ & $7,3(5,4-9,2)$ & $7,6(5,1-10,1)$ \\
\hline & Adultos & $10,5(8,1-13)$ & $9,2(8,2-10,2)$ & $11,0(10,2-11,9)$ & $11,6(10,7-12,4)$ \\
\hline & Idosos & $11,6(7,7-15,4)$ & $10,1(7,9-12,4)$ & $12,1(10,2-14,0)$ & $12,3(11,1-13,6)$ \\
\hline \multicolumn{6}{|l|}{ Zinco (mg) } \\
\hline & Adolescentes & $29,2(26,7-31,7)$ & $25,5(23,6-27,4)$ & $23,2(20,3-26,1)$ & $21,4(17,5-25,3)$ \\
\hline & Adultos & $26,5(24,0-28,9)$ & $23,3(21,6-25,0)$ & $21,0(18,6-23,4)$ & $20,0(17,8-22,2)$ \\
\hline & Idosos & $38,7(34,6-42,7)$ & $34,6(32,5-36,7)$ & $31,3(28,4-34,1)$ & $29,3(27,0-31,7)$ \\
\hline \multicolumn{6}{|c|}{ Vitamina A $(\mathrm{mcg})^{\mathrm{a}}$} \\
\hline & Adolescentes & $89,9(87,3-92,4)$ & $81,5(78,7-84,4)$ & $72,3(66,8-77,9)$ & $55,6(50,9-60,3)$ \\
\hline & Adultos & $93,9(92,9-95,0)$ & $87,2(86,4-88,0)$ & $78,9(77,3-80,5)$ & $64,8(62,2-67,5)$ \\
\hline & Idosos & $92,4(91,4-93,5)$ & $85,0(83,7-86,2)$ & $75,9(74,1-77,8)$ & $60,4(58,4-62,4)$ \\
\hline \multicolumn{6}{|c|}{ Tiamina (mg) } \\
\hline & Adolescentes & $50,3(45,1-55,4)$ & $39,2(37,3-41,0)$ & $35,8(32,4-39,2)$ & $31,2(29,4-32,9)$ \\
\hline & Adultos & $75,3(72,3-78,4)$ & $63,2(61,0-65,4)$ & $58,7(54,9-62,5)$ & $55,3(53,5-57,2)$ \\
\hline & Idosos & $82,0(78,8-85,3)$ & $70,9(69,4-72,3)$ & $66,8(65,0-68,5)$ & $62,5(60,5-64,4)$ \\
\hline \multicolumn{6}{|c|}{ Riboflavina (mg) } \\
\hline & Adolescentes & $50,6(45,6-55,5)$ & $39,5(33,2-45,9)$ & $34,7(26,7-42,7)$ & $28,1(23,9-32,3)$ \\
\hline & Adultos & $66,5(63,6-69,3)$ & $54,6(51,9-57,4)$ & $48,5(44,2-52,8)$ & $42,0(40,4-43,6)$ \\
\hline & Idosos & $65,8(63,3-68,4)$ & $53,3(51,0-55,6)$ & $46,9(43,5-50,2)$ & $39,7(37,6-41,9)$ \\
\hline \multicolumn{6}{|c|}{ Piridoxina (mg) } \\
\hline & Adolescentes & $82,3(80,3-84,4)$ & $75,9(72,6-79,1)$ & $72,7(66,6-78,9)$ & $64,8(62,0-67,7)$ \\
\hline & Adultos & $96,1(95,4-96,7)$ & $93,2(92,0-94,3)$ & $91,4(89,9-92,8)$ & $88,4(87,0-89,7)$ \\
\hline & Idosos & $99,0(98,7-99,2)$ & $98,0(97,5-98,6)$ & $97,2(96,4-98,0)$ & $95,5(94,8-96,2)$ \\
\hline \multicolumn{6}{|c|}{ Cobalamina (mcg) } \\
\hline & Adolescentes & $13,1(9,8-16,3)$ & $13,3(9,5-17,1)$ & $9,9(5,0-14,9)$ & $5,1(2,9-7,3)$ \\
\hline & Adultos & $22,4(20,1-24,7)$ & $22,4(19,9-24,8)$ & $16,9(13,6-20,1)$ & $9,6(8,6-10,5)$ \\
\hline & Idosos & $30,9(26,2-35,6)$ & $30,5(26,1-34,8)$ & $23,5(18,4-28,5)$ & $13,7(12,2-15,2)$ \\
\hline \multicolumn{6}{|l|}{$\begin{array}{l}\text { Vitamina D } \\
\left(\mathrm{mcg}^{\mathrm{b}}\right.\end{array}$} \\
\hline & Adolescentes & $100(99,9-100)$ & $99,9(99,9-100)$ & $99,9(99,8-100)$ & $99,6(99,5-99,7)$ \\
\hline & Adultos & $100(100-100)$ & $100(100-100)$ & $100(100-100)$ & $99,9(99,9-99,9)$ \\
\hline & Idosos & $100(100-100)$ & $100(100-100)$ & $100(100-100)$ & $100(99,9-100)$ \\
\hline \multicolumn{6}{|c|}{ Vitamina $E(m g)^{c}$} \\
\hline & Adolescentes & $94,9(93,2-96,6)$ & $94,1(93,0-95,3)$ & $93,6(90,3-96,8)$ & $91,7(89,4-93,9)$ \\
\hline & Adultos & $98,2(97,6-98,8)$ & $97,5(96,9-98,1)$ & $96,9(95,6-98,1)$ & $95,8(94,7-96,8)$ \\
\hline & Idosos & $98,9(98,4-99,5)$ & $98,7(98,2-99,2)$ & $98,3(97,5-99,0)$ & $97,5(96,8-98,2)$ \\
\hline \multicolumn{6}{|c|}{ Vitamina C (mg) } \\
\hline & Adolescentes & $41,4(33,7-49,1)$ & $28,6(25,7-31,6)$ & $22,9(20,1-25,8)$ & $14,1(12,2-16,1)$ \\
\hline & Adultos & $58,1(53,4-62,8)$ & $43,2(41,5-44,9)$ & $35,6(34,1-37,1)$ & $24,6(23,2-26,0)$ \\
\hline & Idosos & $60,2(54,5-65,9)$ & $45,0(42,1-47,9)$ & $37,4(34,3-40,5)$ & $25,8(23,6-27,9)$ \\
\hline
\end{tabular}

SM: salário-mínimo.

${ }^{a}$ Equivalente de atividade de retinol (RAE).

${ }^{\mathrm{b}}$ Ergocalciterol (D2) + colecalciterol (D3).

${ }^{c}$ Total de alfa-tocoferol. 
Tabela 7. Prevalências de inadequação (IC95\%) da ingestão de vitaminas e minerais segundo região entre homens no Inquérito Nacional de Alimentação (INA/POF) 2008-2009.

\begin{tabular}{|c|c|c|c|c|c|c|}
\hline \multirow{3}{*}{ Nutrientes } & \multirow{3}{*}{ Faixa etária } & \multicolumn{5}{|c|}{ Região } \\
\hline & & Norte & Nordeste & Sul & Sudeste & Centro-oeste \\
\hline & & $\%($ (IC95\%) & $\%($ IC95\%) & $\%($ IC95\%) & $\%($ (IC95\%) & $\%($ IC95\%) \\
\hline \multicolumn{7}{|l|}{ Cálcio (mg) } \\
\hline & Adolescentes & $96,8(96,3-97,3)$ & $98,6(98,2-99,0)$ & $96,8(95,8-97,7)$ & $96,5(95,7-97,3)$ & $98,5(98,1-98,8)$ \\
\hline & Adultos & $87,5(86,4-88,5)$ & $93,3(92,7-93,9)$ & $87,1(84,8-89,4)$ & $86,7(85,7-87,8)$ & $92,6(92,1-93,0)$ \\
\hline & Idosos & $91,0(89,7-92,2)$ & $95,6(94,8-96,5)$ & $90,8(88,3-93,3)$ & $90,7(89,1-92,3)$ & $95,0(94,1-96,0)$ \\
\hline \multicolumn{7}{|c|}{ Magnésio (mg) } \\
\hline & Adolescentes & $47,5(42,9-52,2)$ & $56,5(54,3-58,7)$ & $46,6(45,1-48,1)$ & $52,0(48,5-55,4)$ & $46,5(41,8-51,1)$ \\
\hline & Adultos & $64,6(63,0-66,2)$ & $72,8(70,9-74,7)$ & $62,0(60,4-63,6)$ & $68,1(65,9-70,2)$ & $65,1(63,6-66,7)$ \\
\hline & Idosos & $75,5(70,6-80,4)$ & $82,7(78,6-86,7)$ & $73,4(71,4-75,5)$ & $78,7(74,5-82,9)$ & $76,1(72,4-79,9)$ \\
\hline \multicolumn{7}{|l|}{ Fósforo (mg) } \\
\hline & Adolescentes & $44,4(41,8-47,1)$ & $55,0(52,2-57,8)$ & $46,7(44,7-48,7)$ & $52,0(48,8-55,2)$ & $56,0(52,5-59,5)$ \\
\hline & Adultos & $1,7(1,5-2,0)$ & $3,5(3,1-3,9)$ & $2,4(1,7-3,1)$ & $3,1(2,6-3,7)$ & $3,6(3,1-4,1)$ \\
\hline & Idosos & $4,6(3,7-5,6)$ & $8,3(6,6-9,9)$ & $5,2(4,5-5,9)$ & $7,0(5,6-8,3)$ & $8,4(6,8-10,1)$ \\
\hline \multicolumn{7}{|l|}{ Cobre (mg) } \\
\hline & Adolescentes & $3,6(2,8-4,5)$ & $3,5(3,0-4,0)$ & $2,5(2,2-2,8)$ & $3,9(2,7-5,1)$ & $2,4(2,0-2,9)$ \\
\hline & Adultos & $4,4(3,5-5,2)$ & $4,3(3,8-4,9)$ & $2,9(2,6-3,3)$ & $4,7(3,7-5,8)$ & $2,7(2,4-3,1)$ \\
\hline & Idosos & $7,6(5,5-9,7)$ & $7,8(5,6-10,0)$ & $4,9(3,7-6,2)$ & $8,2(5,3-11,1)$ & $4,8(3,1-6,6)$ \\
\hline \multicolumn{7}{|l|}{ Zinco (mg) } \\
\hline & Adolescentes & $14,1(10,6-17,5)$ & $16,7(13,8-19,6)$ & $17,9(15,3-20,4)$ & $15,7(11,1-20,2)$ & $12,4(9,6-15,3)$ \\
\hline & Adultos & $19,1(17,0-21,2)$ & $22,4(21,2-23,7)$ & $24,3(23,2-25,3)$ & $20,9(16,8-25,0)$ & $17,2(15,5-18,9)$ \\
\hline & Idosos & $29,4(26,6-32,2)$ & $33,9(30,6-37,2)$ & $35,0(31,7-38,4)$ & $31,9(26,6-37,2)$ & $27,5(24,6-30,5)$ \\
\hline \multicolumn{7}{|c|}{ Vitamina A $(\mathrm{mcg})^{\mathrm{a}}$} \\
\hline & Adolescentes & $83,1(80,1-86,2)$ & $83,7(80,6-86,7)$ & $75,2(72,2-78,1)$ & $74,6(70,2-79,0)$ & $79,1(75,4-82,7)$ \\
\hline & Adultos & $89,0(87,3-90,7)$ & $89,0(87,5-90,5)$ & $82,0(80,4-83,6)$ & $81,5(78,6-84,5)$ & $86,1(84,4-87,8)$ \\
\hline & Idosos & $89,2(87,2-91,2)$ & $89,6(87,4-91,7)$ & $82,1(80,4-83,9)$ & $82,3(79,9-84,8)$ & $86,5(84,4-88,6)$ \\
\hline \multicolumn{7}{|c|}{ Tiamina (mg) } \\
\hline & Adolescentes & $49,9(47,0-52,7)$ & $48,2(43,0-53,5)$ & $35,3(33,1-37,5)$ & $32,0(28,8-35,1)$ & $48,0(44,9-51,2)$ \\
\hline & Adultos & $69,5(67,0-71,9)$ & $67,6(63,6-71,5)$ & $54,7(49,9-59,6)$ & $51,3(49,1-53,4)$ & $68,8(65,6-72,0)$ \\
\hline & Idosos & $78,9(76,4-81,4)$ & $77,7(74,0-81,4)$ & $65,4(63,6-67,3)$ & $62,2(57,9-66,5)$ & $78,3(75,0-81,6)$ \\
\hline \multicolumn{7}{|c|}{ Riboflavina (mg) } \\
\hline & Adolescentes & $38,9(34,2-43,6)$ & $34,7(27,6-41,7)$ & $28,1(24,6-31,6)$ & $25,7(21,3-30,2)$ & $36,0(31,6-40,3)$ \\
\hline & Adultos & $54,9(51,4-58,4)$ & $49,9(44,2-55,5)$ & $42,4(40,6-44,3)$ & $40,2(36,3-44,2)$ & $53,3(49,5-57,1)$ \\
\hline & Idosos & $57,4(53,8-61,0)$ & $53,0(47,8-58,3)$ & $44,4(42,2-46,7)$ & $42,8(37,6-47,9)$ & $56,8(53,1-60,4)$ \\
\hline \multicolumn{7}{|c|}{ Piridoxina (mg) } \\
\hline & Adolescentes & $75,9(73,4-78,4)$ & $75,2(71,4-78,9)$ & $66,4(63,2-69,7)$ & $62,4(59,8-65,0)$ & $76,6(73,0-80,1)$ \\
\hline & Adultos & $85,9(82,8-89,0)$ & $85,2(81,8-88,5)$ & $79,2(73,7-84,8)$ & $76,2(73,5-78,9)$ & $87,2(83,6-90,7)$ \\
\hline & Idosos & $97,6(96,7-98,5)$ & $97,3(96,2-98,3)$ & $94,9(92,8-97,0)$ & $94,1(93,0-95,1)$ & $97,8(96,8-98,8)$ \\
\hline \multicolumn{7}{|c|}{ Cobalamina (mcg) } \\
\hline & Adolescentes & $1,0(0,5-1,5)$ & $5,8(3,9-7,7)$ & $7,5(5,4-9,6)$ & $6,8(3,5-10,1)$ & $5,5(3,6-7,5)$ \\
\hline & Adultos & $0,7(0,4-1,0)$ & $5,0(3,9-6,0)$ & $6,4(5,0-7,8)$ & $5,7(3,7-7,8)$ & $4,5(3,6-5,5)$ \\
\hline & Idosos & $1,2(0,6-1,8)$ & $7,5(6,1-8,9)$ & $8,5(6,3-10,8)$ & $8,0(5,5-10,6)$ & $6,6(5,0-8,3)$ \\
\hline \multicolumn{7}{|c|}{ Vitamina D $(\mathrm{mcg})^{\mathrm{b}}$} \\
\hline & Adolescentes & $98,5(97,9-99,0)$ & $99,5(99,3-99,6)$ & $99,4(99,2-99,6)$ & $99,5(99,2-99,8)$ & $99,6(99,3-99,8)$ \\
\hline & Adultos & $98,9(98,4-99,4)$ & $99,6(99,5-99,8)$ & $99,6(99,4-99,9)$ & $99,7(99,5-99,9)$ & $99,7(99,5-99,9)$ \\
\hline & Idosos & $99,1(98,6-99,7)$ & $99,8(99,6-99,9)$ & $99,7(99,6-99,9)$ & $99,8(99,6-100)$ & $99,8(99,7-99,9)$ \\
\hline \multicolumn{7}{|c|}{ Vitamina $\mathrm{E}(\mathrm{mg})^{\mathrm{c}}$} \\
\hline & Adolescentes & $81,0(78,6-83,3)$ & $93,8(92,9-94,6)$ & $89,5(88,4-90,6)$ & $92,5(91,1-93,9)$ & $90,9(89,5-92,3)$ \\
\hline & Adultos & $86,1(84,0-88,3)$ & $96,1(95,4-96,7)$ & $92,8(92,0-93,5)$ & $95,3(94,2-96,4)$ & $94,2(93,4-95,0)$ \\
\hline & Idosos & $90,7(88,6-92,7)$ & $97,6(97,3-98,0)$ & $95,3(94,4-96,2)$ & $97,2(96,7-97,7)$ & $96,6(96,1-97,1)$ \\
\hline \multicolumn{7}{|c|}{ Vitamina C (mg) } \\
\hline & Adolescentes & $34,8(32,2-37,4)$ & $41,2(38,5-43,8)$ & $30,1(28,6-31,6)$ & $25,4(22,5-28,2)$ & $27,9(25,7-30,1)$ \\
\hline & Adultos & $46,7(42,9-50,5)$ & $52,9(50,5-55,3)$ & $40,9(38,8-43,1)$ & $35,7(33,6-37,7)$ & $39,7(37,8-41,6)$ \\
\hline & Idosos & $48,5(44,4-52,6)$ & $55,1(52,1-58,2)$ & $42,3(39,1-45,4)$ & $37,7(33,4-42,0)$ & $42,0(39,1-44,8)$ \\
\hline
\end{tabular}

a Equivalente de atividade de retinol (RAE).

${ }^{\mathrm{b}}$ Ergocalciterol (D2) + colecalciterol (D3).

${ }^{c}$ Alfa-tocoferol total. 
Tabela 8. Prevalências de inadequação (IC95\%) da ingestão de vitaminas e minerais segundo região entre mulheres no Inquérito Nacional de Alimentação (INA/POF) 2008-2009.

\begin{tabular}{|c|c|c|c|c|c|c|}
\hline \multirow{3}{*}{ Nutrientes } & \multirow{3}{*}{ Faixa etária } & \multicolumn{5}{|c|}{ Região } \\
\hline & & Norte & Nordeste & Sul & Sudeste & Centro-oeste \\
\hline & & $\%$ (IC95\%) & $\%$ (IC95\%) & $\%$ (IC95\%) & $\%($ (IC95\%) & $\%$ (IC95\%) \\
\hline
\end{tabular}

\section{Cálcio $(\mathrm{mg})$}

Adolescentes $\quad 97,7(97,3-98,2) \quad 99,0(98,7-99,2)$

Adultos $\quad 92,3(91,7-92,9) \quad 96,2(95,8-96,7)$

Idosos $\quad 97,1(96,4-97,8)$

Magnésio (mg)

Adolescentes
Adultos
Idosos

$54,2(51,6-56,9)$
$62,4(60,6-64,3)$
$67,5(65,8-69,1)$

$98,7(98,5-98,9)$

$64,1(61,2-67,0)$
$71,0(69,3-72,6)$
$75,3(73,6-77,0)$

Adolescentes
Adultos
Idosos

$58,1(55,9-60,2)$
$8,4(7,8-9,0)$
$11,6(10,7-12,5)$

$68,3(66,7-69,8)$

$18,0(16,4-19,5)$

Cobre (mg)

Adolescentes
Adultos
Idosos

$8,0(6,9-9,1)$

$15,0(13,5-16,6)$

$17,3(15,3-19,3)$

$97,4(97,1-97,7)$
$92,6(91,3-93,8)$
$97,1(96,3-97,9)$

$54,2(47,7-60,8)$
$59,9(58,7-61,2)$
$64,9(63,6-66,3)$

$97,2(96,7-97,8)$
$92,0(90,5-93,4)$
$96,7(95,9-97,5)$

$98,7(98,4-99,0)$

$96,1(95,6-96,6)$

$98,3(98,0-98,6)$

$58,8(55,0-62,7)$

$56,6(53,0-60,2)$

$65,4(64,0-66,9)$

$63,1(61,3-64,9)$

$69,5(66,9-72,1)$

$67,8(66,0-69,5)$

Zinco (mg)

\begin{tabular}{|c|c|c|c|c|c|c|}
\hline & Adolescentes & $18,1(15,4-20,8)$ & $21,2(18,8-23,6)$ & $23,1(20,7-25,4)$ & $19,9(15,2-24,7)$ & $16,7(14,2-19,1)$ \\
\hline & Adultos & $15,1(13,3-16,9)$ & $18,1(17,3-18,8)$ & $19,9(19,1-20,7)$ & $16,7(13,1-20,4)$ & $13,7(12,2-15,1)$ \\
\hline & Idosos & $19,6(16,1-23,1)$ & $23,1(19,9-26,3)$ & $24,4(21,7-27,2)$ & $21,1(17,3-24,9)$ & $17,6(14,7-20,4)$ \\
\hline \multicolumn{7}{|l|}{ Vitamina A (mcg) ${ }^{\mathrm{a}}$} \\
\hline & Adolescentes & $75,4(71,6-79,2)$ & $75,5(71,3-79,7)$ & $64,7(61,1-68,3)$ & $63,8(59,2-68,5)$ & $70,3(66,1-74,5)$ \\
\hline & Adultos & $78,6(75,5-81,7)$ & $78,8(76,2-81,4)$ & $68,8(66,3-71,3)$ & $67,8(64,1-71,4)$ & $74,4(71,3-77,4)$ \\
\hline & Idosos & $75,5(71,5-79,4)$ & $74,8(72,1-77,6)$ & $64,2(61,9-66,6)$ & $62,6(59,3-65,9)$ & $70,7(67,7-73,7)$ \\
\hline \multicolumn{7}{|l|}{ Tiamina (mg) } \\
\hline & Adolescentes & $51,4(47,8-55,1)$ & $49,9(44,8-55,0)$ & $36,6(34,3-39,0)$ & $32,8(30,4-35,1)$ & $51,2(46,1-56,4)$ \\
\hline & Adultos & $73,0(71,2-74,7)$ & $71,2(67,8-74,6)$ & $58,9(56,9-60,9)$ & $54,5(53,5-55,4)$ & $72,3(69,5-75,1)$ \\
\hline & Idosos & $80,4(77,9-82,9)$ & $77,9(74,9-81,0)$ & $66,5(62,5-70,4)$ & $61,9(60,3-63,6)$ & $79,1(77,1-81,1)$ \\
\hline \multicolumn{7}{|l|}{ Riboflavina (mg) } \\
\hline & Adolescentes & $34,4(29,1-39,7)$ & $30,2(22,3-38,2)$ & $23,7(20,4-27,0)$ & $21,6(17,0-26,2)$ & $33,1(27,2-39,1)$ \\
\hline & Adultos & $45,6(42,0-49,3)$ & $40,8(34,9-46,6)$ & $33,5(32,3-34,6)$ & $30,6(26,9-34,3)$ & $43,7(40,2-47,2)$ \\
\hline & Idosos & $46,5(40,5-52,5)$ & $41,7(35,6-47,7)$ & $33,7(30,9-36,5)$ & $31,1(26,0-36,2)$ & $44,4(40,1-48,7)$ \\
\hline \multicolumn{7}{|l|}{ Piridoxina (mg) } \\
\hline & Adolescentes & $76,9(72,9-80,9)$ & $76,2(72,1-80,3)$ & $67,6(62,1-73,0)$ & $63,2(61,3-65,1)$ & $78,3(72,1-84,5)$ \\
\hline & Adultos & $92,4(90,5-94,3)$ & $92,1(90,0-94,2)$ & $88,2(85,4-91,0)$ & $85,5(83,7-87,3)$ & $93,5(91,4-95,5)$ \\
\hline & Idosos & $98,0(96,9-99,2)$ & $97,6(96,6-98,6)$ & $96,0(94,2-97,8)$ & $94,7(93,3-96,0)$ & $98,0(97,2-98,7)$ \\
\hline \multicolumn{7}{|l|}{ Cobalamina (mcg) } \\
\hline & Adolescentes & $1,4(0,5-2,2)$ & $7,4(4,0-10,8)$ & $9,0(4,9-13,0)$ & $8,3(3,7-12,9)$ & $7,2(4,0-10,3)$ \\
\hline & Adultos & $2,0(1,3-2,6)$ & $10,1(8,7-11,5)$ & $12,5(10,3-14,8)$ & $11,3(8,3-14,3)$ & $9,6(8,2-11,0)$ \\
\hline & Idosos & $2,3(1,6-3,1)$ & $11,6(9,8-13,3)$ & $13,5(11,8-15,3)$ & $12,8(10,7-14,9)$ & $10,8(9,0-12,7)$ \\
\hline \multicolumn{7}{|l|}{ Vitamina D $(\mathrm{mcg})^{\mathrm{b}}$} \\
\hline & Adolescentes & $98,8(98,5-99,2)$ & $99,5(99,4-99,7)$ & $99,5(99,3-99,7)$ & $99,7(99,5-99,8)$ & $99,7(99,6-99,8)$ \\
\hline & Adultos & $99,4(99,1-99,7)$ & $99,8(99,7-99,9)$ & $99,8(99,7-99,9)$ & $99,8(99,7-99,9)$ & $99,9(99,8-99,9)$ \\
\hline & Idosos & $99,5(99,2-99,8)$ & $99,8(99,7-100)$ & $99,8(99,6-100)$ & $99,9(99,8-100)$ & $99,9(99,7-100)$ \\
\hline \multicolumn{7}{|l|}{ Vitamina $E(m g)^{c}$} \\
\hline & Adolescentes & $87,0(84,4-89,6)$ & $96,2(95,5-96,9)$ & $93,4(92,3-94,5)$ & $95,5(94,3-96,6)$ & $94,5(93,5-95,4)$ \\
\hline & Adultos & $95,0(93,8-96,1)$ & $99,0(98,8-99,1)$ & $97,9(97,6-98,2)$ & $98,6(98,3-99,0)$ & $98,5(98,2-98,7)$ \\
\hline & Idosos & $95,9(94,7-97,0)$ & $99,1(98,9-99,4)$ & $98,2(98,0-98,5)$ & $98,9(98,7-99,0)$ & $98,5(98,2-98,8)$ \\
\hline \multicolumn{7}{|l|}{ Vitamina C (mg) } \\
\hline & Adolescentes & $30,8(26,2-35,4)$ & $36,9(34,7-39,1)$ & $26,1(21,5-30,6)$ & $21,5(18,9-24,1)$ & $25,4(21,8-29,1)$ \\
\hline & Adultos & $37,1(33,2-41,0)$ & $43,4(40,7-46,0)$ & $31,9(30,7-33,0)$ & $26,5(24,4-28,7)$ & $30,6(28,5-32,7)$ \\
\hline & Idosos & $37,4(32,2-42,5)$ & $43,9(41,2-46,6)$ & $31,9(27,8-36,1)$ & $26,7(23,7-29,7)$ & $30,9(27,9-34,0)$ \\
\hline
\end{tabular}

a Equivalente de atividade de retinol (RAE).

${ }^{\mathrm{b}}$ Ergocalciterol (D2) + colecalciterol (D3).

c Alfa-tocoferol total.
$13,6(12,8-14,4)$

$8,4(7,4-9,4)$

$15,0(13,9-16,1)$

$17,4(16,2-18,5)$

$61,0(59,3-62,7)$

$10,1(9,2-11,0)$

$65,8(62,8-68,7)$

$12,2(10,9-13,5)$

$13,1(12,3-13,8)$

$15,9(15,0-16,8)$

$68,5(66,5-70,4)$

$14,0(13,1-15,0)$

$18,1(16,9-19,2)$

$6,0(5,1-6,8)$
$11,1(10,0-12,2)$
$12,7(11,5-14,0)$

$9,2(7,0-11,4)$

$6,1(4,8-7,4)$

$16,0(13,6-18,3)$

$10,9(9,6-12,2)$

$18,2(15,9-20,6)$

$12,8(11,3-14,3)$

$23,1(20,7-25,4)$

$19,9(19,1-20,7$

$24,4(21,7-27,2)$

$19,9(15,2-24,7)$

$16,7(14,2-19,1)$

$13,7(12,2-15,1)$

$16,7(13,1-20,4)$

(14,7-20,4)

$64,7(61,1-68,3)$

$68,8(66,3-71,3)$

$67,8(64,1-71,4)$

$74,4(71,3-77,4)$

$74,8(72,1-77,6)$

$36,6(34,3-39,0)$

$32,8(30,4-35,1)$

$51,2(46,1-56,4)$

$58,9(56,9-60,9)$

54,5 (53,5-55,4)

$2,3(69,5-75,1)$

$71,2(67,8-74,6)$

$66,5(62,5-70,4)$

$21,6(17,0-26,2)$

$33,1(27,2-39,1)$

$33,5(32,3-34,6)$

$0,6(26,9-34,3)$

$78,3(72,1-84,5)$

$93,5(91,4-95,5)$

$88,2(85,4-91,0)$

$85,5(83,7-87,3)$

$94,7(93,3-96,0)$

$$
8,3(3,7-12,9)
$$

$7,2(4,0-10,3)$

(8,2-11,0)

$10,1(8,7-11,5)$

$99,5(99,3-99,7)$
$99,8(99,7-99,9)$
$99,8(99,6-100)$

99,8 $(99,7-99,9)$

$99,9(99,8-99,9)$

$99,9(99,7-100)$

$94,5(93,5-95,4)$

$98,5(98,2-98,7)$

$97,9(97,6-98,2$

$98,6(98,3-99,0)$

$98,9(98,7-99,0)$

$98,5(98,2-98,8)$
$43,7(40,2-47,2)$

$30,9(27,9-34,0)$ 
No último inquérito, excetuando as estimativas relativas a cobre, magnésio e vitamina $\mathrm{D}$ entre idosos, e vitamina $\mathrm{E}$ entre mulheres adolescentes, diferenças significantes foram observadas entre os dois extremos de renda per capita ( $<0,5 v s .>2$ salários-mínimos), sendo as mais expressivas ( $>15$ pontos percentuais) para vitamina $\mathrm{C}$, vitamina $\mathrm{A}$, riboflavina $\mathrm{e}$ tiamina em todas as idades, fósforo e piridoxina entre adolescentes, e cálcio entre homens adultos (Tabelas 5 e 6 ).

Quando as análises foram estratificadas pelas regiões do país, resultados semelhantes foram encontrados para homens e mulheres, tanto em 2008-2009 (Tabelas 7 e 8) quanto em 2017-2018 (Tabelas 9 e 10). Verificou-se que tiamina, zinco, vitamina C, magnésio, cobalamina e cobre se destacaram como os micronutrientes mais variáveis entre as

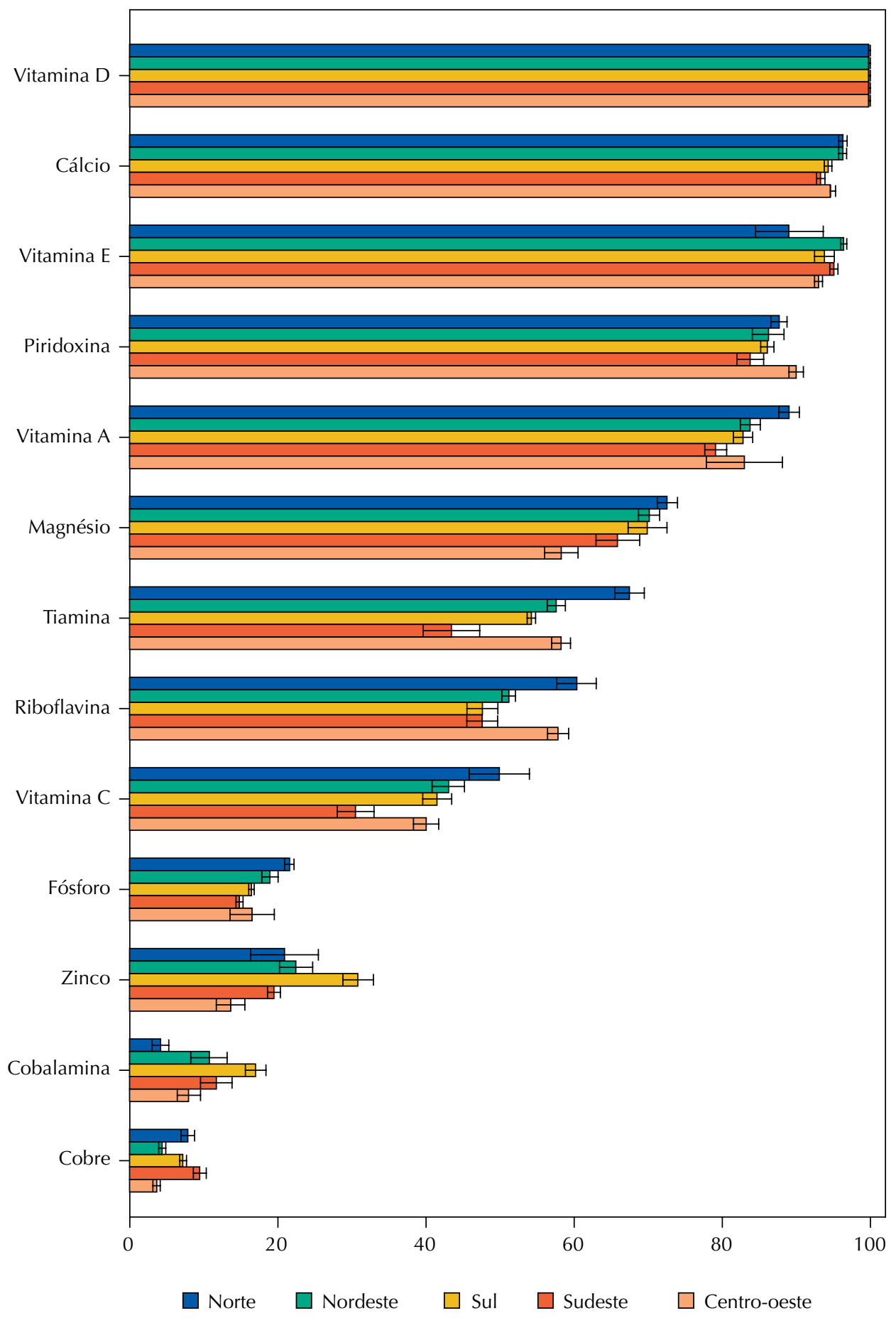

Figura 1. Prevalências de inadequação (IC95\%) da ingestão de vitaminas e minerais segundo região no Inquérito Nacional de Alimentação (INA/POF) 2017-2018. 
regiões (Figuras 1 e 2). Em 2017-2018, as regiões Norte e Nordeste, seguidas por Centro-Oeste, destacaram-se entre a primeira e a segunda posições de maior prevalência de inadequação para a maioria dos nutrientes analisados. Entre as diferenças estatisticamente significativas, optou-se por destacar aquelas mais evidentes ( $>15$ pontos percentuais).

Discrepâncias foram encontradas nas prevalências de inadequação de tiamina entre Norte e Sudeste e de zinco entre Sul e Centro-Oeste, cujas estimativas, em todas as faixas etárias, foram de 1,5 a 2 vezes maiores na primeira região em relação à última, respectivamente. A região Sudeste se destacou com menores prevalências de inadequação de tiamina em relação às regiões Nordeste e Centro-Oeste entre adultos e idosos, ao passo que o Sul, na segunda posição de menor inadequação, ainda diferiu em relação

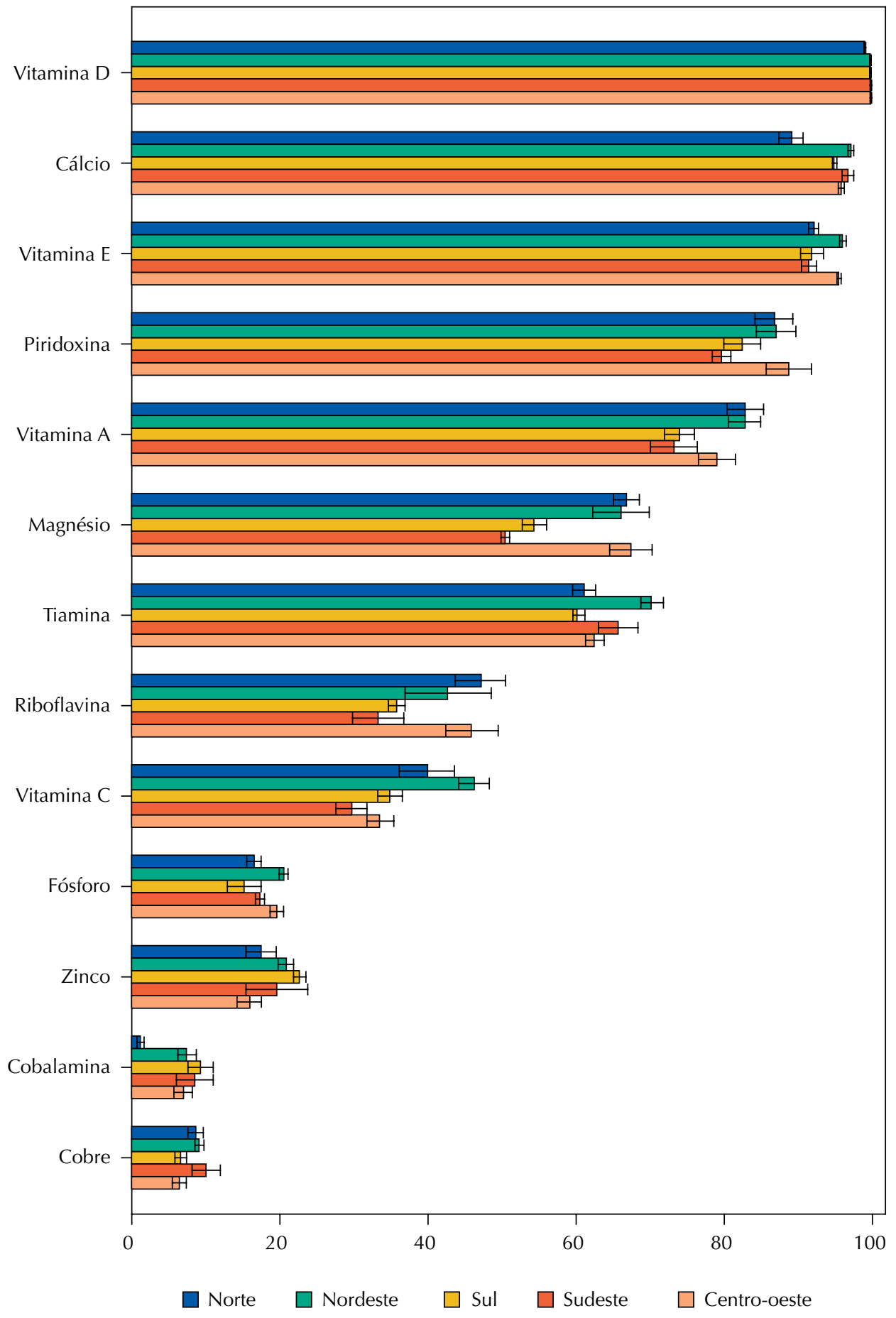

Figura 2. Prevalências de inadequação (IC95\%) da ingestão de vitaminas e minerais segundo região no Inquérito Nacional de Alimentação (INA/POF) 2008-2009. 
Tabela 9. Prevalências de inadequação (IC95\%) da ingestão de vitaminas e minerais segundo região entre homens no Inquérito Nacional de Alimentação (INA/POF) 2017-2018.

\begin{tabular}{|c|c|c|c|c|c|c|}
\hline \multirow{3}{*}{ Nutrientes } & \multirow{3}{*}{ Faixa etária } & \multicolumn{5}{|c|}{ Região } \\
\hline & & Norte & Nordeste & Sul & Sudeste & Centro-oeste \\
\hline & & $\%($ IC95\%) & $\%($ IC95\%) & $\%($ IC95\%) & $\%($ IC95\%) & $\%($ IC95\%) \\
\hline \multicolumn{7}{|l|}{ Cálcio (mg) } \\
\hline & Adolescentes & $98,8(98,5-99,1)$ & $98,6(98,4-98,9)$ & $97,9(97,4-98,3)$ & $97,3(96,9-97,6)$ & $98,0(97,7-98,4)$ \\
\hline & Adultos & $93,3(92,1-94,5)$ & $93,2(92,3-94,0)$ & $89,9(88,6-91,3)$ & $88,5(87,4-89,7)$ & $91,1(90,5-91,7)$ \\
\hline & Idosos & $96,2(94,8-97,5)$ & $96,1(95,0-97,2)$ & $93,8(93,4-94,3)$ & $92,7(91,3-94,1)$ & $94,4(93,4-95,4)$ \\
\hline \multicolumn{7}{|c|}{ Magnésio (mg) } \\
\hline & Adolescentes & $58,4(56,6-60,2)$ & $55,9(53,9-57,9)$ & $54,0(49,7-58,4)$ & $53,4(46,7-60,2)$ & $43,7(40,3-47,1)$ \\
\hline & Adultos & $74,4(73,1-75,6)$ & $71,1(69,3-72,9)$ & $70,2(67,7-72,6)$ & $65,8(63,3-68,3)$ & $58,9(56,5-61,2)$ \\
\hline & Idosos & $84,8(83,1-86,6)$ & $82,3(80,4-84,3)$ & $81,3(79,1-83,5)$ & $77,8(75,0-80,6)$ & $71,8(70,1-73,6)$ \\
\hline \multicolumn{7}{|c|}{ Fósforo (mg) } \\
\hline & Adolescentes & $56,1(54,3-58,0)$ & $52,9(50,4-55,3)$ & $51,2(48,0-54,3)$ & $48,3(46,5-50,2)$ & $50,6(45,3-55,8)$ \\
\hline & Adultos & $2,7(2,0-3,4)$ & $2,3(1,7-2,8)$ & $2,13(2,0-2,3)$ & $1,78(1,3-2,3)$ & $1,87(1,1-2,7)$ \\
\hline & Idosos & $8,5(7,0-10,0)$ & $7,4(6,1-8,7)$ & $7,0(6,6-7,3)$ & $5,81(5,0-6,7)$ & $6,57(4,8-8,3)$ \\
\hline \multicolumn{7}{|l|}{ Cobre (mg) } \\
\hline & Adolescentes & $3,0(2,6-3,4)$ & $1,3(1,2-1,4)$ & $2,3(1,8-2,8)$ & $3,5(2,7-4,4)$ & $1,0(0,8-1,2)$ \\
\hline & Adultos & $3,3(2,7-3,9)$ & $1,4(1,2-1,7)$ & $2,6(2,2-3,0)$ & $3,7(3,1-4,3)$ & $1,1(0,8-1,4)$ \\
\hline & Idosos & $5,8(4,6-7,1)$ & $2,7(2,2-3,2)$ & $4,9(4,3-5,4)$ & $6,5(5,8-7,2)$ & $2,3(1,9-2,8)$ \\
\hline \multicolumn{7}{|l|}{ Zinco (mg) } \\
\hline & Adolescentes & $15,5(12,3-18,8)$ & $16,1(12,8-19,3)$ & $22,5(20,3-24,8)$ & $13,7(10,6-16,8)$ & $9,2(6,6-11,8)$ \\
\hline & Adultos & $20,5(16,1-2)$ & $21,4(19,1-23,6)$ & $29,3(27,1-31,5)$ & $18,0(17,2-18,9)$ & $12,8(10,8-14,9)$ \\
\hline & Idosos & $34,7(28-41,4)$ & $36,3(33,3-39,2)$ & $45,7(44,5-46,8)$ & $31,6(30,1-33,1)$ & $24,3(21,7-26,9)$ \\
\hline \multicolumn{7}{|c|}{ Vitamina A $(\mathrm{mcg})^{\mathrm{a}}$} \\
\hline & Adolescentes & $88,7(86,2-91,1)$ & $84,0(81,2-86,8)$ & $82,9(81,4-84,4)$ & $80,1(76,9-83,3)$ & $83,0(77,3-88,7)$ \\
\hline & Adultos & $93,5(92,6-94,5)$ & $89,9(88,8-91,1)$ & $89,1(87,9-90,4)$ & $86,1(84,9-87,3)$ & $89,1(84,8-93,5)$ \\
\hline & Idosos & $92,2(90,6-93,8)$ & $88,3(86,1-90,5)$ & $87,6(86,1-89,2)$ & $84,2(82,4-86,0)$ & $87,6(83,4-91,8)$ \\
\hline \multicolumn{7}{|c|}{ Tiamina (mg) } \\
\hline & Adolescentes & $45,2(41,8-48,5)$ & $34,1(31,1-37,0)$ & $29,1(27,1-31,2)$ & $22,4(18,2-26,6)$ & $33,6(30,8-36,3)$ \\
\hline & Adultos & $67,2(65,0-69,3)$ & $55,2(54,1-56,2)$ & $50,2(49,4-51,1)$ & $39,3(35,3-43,3)$ & $55,7(54,3-57,2)$ \\
\hline & Idosos & $78,2(76,1-80,4)$ & $67,4(65,1-69,8)$ & $63,2(61,6-64,9)$ & $51,4(47,4-55,4)$ & $67,6(65,4-69,9)$ \\
\hline \multicolumn{7}{|c|}{ Riboflavina (mg) } \\
\hline & Adolescentes & $45,8(43,1-48,4)$ & $37,2(34,5-39,8)$ & $32,8(29,7-35,8)$ & $34,6(31,6-37,7)$ & $42,3(40,5-44,0)$ \\
\hline & Adultos & $65,6(62,7-68,5)$ & $56,1(54,1-58,2)$ & $51,9(50,8-53,0)$ & $51,7(49,1-54,3)$ & $62,4(60,4-64,4)$ \\
\hline & Idosos & $68,7(65,8-71,6)$ & $59,1(57,3-60,9)$ & $55,7(54,5-57,0)$ & $54,6(52,1-57,1)$ & $65,7(63,3-68,0)$ \\
\hline \multicolumn{7}{|c|}{ Piridoxina (mg) } \\
\hline & Adolescentes & $73,5(71,0-76,0)$ & $69,5(64,9-74,0)$ & $66,8(63,6-70,0)$ & $65,1(61,0-69,2)$ & $75,7(73,8-77,7)$ \\
\hline & Adultos & $85,7(84,1-87,2)$ & $82,8(79,4-86,1)$ & $81,6(80,6-82,6)$ & $78,7(76,0-81,4)$ & $87,5(85,8-89,2)$ \\
\hline & Idosos & $96,7(96,0-97,4)$ & $95,5(94,5-96,5)$ & $94,8(94,1-95,5)$ & $93,3(92,3-94,3)$ & $97,1(96,6-97,6)$ \\
\hline \multicolumn{7}{|c|}{ Cobalamina $(\mathrm{mcg})$} \\
\hline & Adolescentes & $2,6(2,2-3,0)$ & $6,8(5,3-8,3)$ & $10,8(9,4-12,1)$ & $7,7(5,4-10,0)$ & $4,8(4,0-5,6)$ \\
\hline & Adultos & $2,1(1,5-2,7)$ & $5,8(4,4-7,2)$ & $9,9(9,0-10,9)$ & $6,3(4,9-7,6)$ & $4,1(3,3-5,0)$ \\
\hline & Idosos & $4,3(2,8-5,8)$ & $10,9(8,0-13,8)$ & $17,1(15,8-18,5)$ & $11,5(9,6-13,4)$ & $8,2(6,7-9,8)$ \\
\hline \multicolumn{7}{|c|}{ Vitamina D (mcg) $)^{b}$} \\
\hline & Adolescentes & $99,8(99,7-99,9)$ & $99,9(99,8-99,9)$ & $99,8(99,7-99,9)$ & $99,8(99,8-99,9)$ & $99,8(99,7-99,9)$ \\
\hline & Adultos & $99,9(99,8-99,9)$ & $99,9(99,9-100)$ & $99,9(99,9-100)$ & $99,9(99,8-99,9)$ & $99,9(99,8-100)$ \\
\hline & Idosos & $100(99,9-100)$ & $100(100-100)$ & $100(99,9-100)$ & $99,9(99,9-100)$ & $99,9(99,9-100)$ \\
\hline \multicolumn{7}{|c|}{ Vitamina $E(m g)^{c}$} \\
\hline & Adolescentes & $81,8(76,3-87,2)$ & $92,8(91,9-93,8)$ & $88,0(85,3-90,7)$ & $90,6(89,5-91,6)$ & $87,4(86,4-88,5)$ \\
\hline & Adultos & $85,3(79,3-91,3)$ & $94,7(94,1-95,4)$ & $90,7(89,2-92,2)$ & $92,7(91,8-93,6)$ & $90,0(89,1-91,0)$ \\
\hline & Idosos & $91,2(86,3-96,1)$ & $97,3(96,7-97,8)$ & $94,7(93,5-96,0)$ & $95,7(95,0-96,4)$ & $94,2(93,3-95,1)$ \\
\hline \multicolumn{7}{|c|}{ Vitamina C (mg) } \\
\hline & Adolescentes & $42,7(38,0-47,3)$ & $36,2(31,6-40,8)$ & $33,9(29,2-38,6)$ & $25,3(20,5-30,1)$ & $32,8(28,5-37,0)$ \\
\hline & Adultos & $57,2(53,8-60,5)$ & $50,0(48,2-51,9)$ & $48,0(45,7-50,3)$ & $36,1(33,2-38,9)$ & $46,6(44,9-48,4)$ \\
\hline & Idosos & $58,8(53,4-64,2)$ & $51,4(48,1-54,8)$ & $50,1(48,0-52,1)$ & $37,6(34,0-41,2)$ & $48,6(45,5-51,7)$ \\
\hline
\end{tabular}

a Equivalente de atividade de retinol (RAE).

${ }^{\mathrm{b}}$ Ergocalciterol (D2) + colecalciterol (D3).

c Alfa-tocoferol total. 
Tabela 10. Prevalências de inadequação (IC95\%) da ingestão de vitaminas e minerais segundo região entre mulheres no Inquérito Nacional de Alimentação (INA/POF) 2017-2018.

\begin{tabular}{|c|c|c|c|c|c|c|}
\hline \multirow{3}{*}{ Nutrientes } & \multirow{3}{*}{ Faixa etária } & \multicolumn{5}{|c|}{ Região } \\
\hline & & Norte & Nordeste & Sul & Sudeste & Centro-oeste \\
\hline & & $\%($ IC95\%) & $\%($ IC95\%) & $\%($ IC95\%) & $\%($ IC95\%) & $\%($ IC95\%) \\
\hline \multicolumn{7}{|l|}{ Cálcio (mg) } \\
\hline & Adolescentes & $99,4(99,0-99,8)$ & $99,3(98,8-99,7)$ & $98,8(98,4-99,1)$ & $98,5(97,8-99,3)$ & $99,0(98,4-99,6)$ \\
\hline & Adultos & $97,2(96,5-97,8)$ & $97,2(96,5-97,8)$ & $95,7(95,5-95,9)$ & $95,0(94,2-95,8)$ & $96,3(95,6-96,9)$ \\
\hline & Idosos & $99,1(98,8-99,4)$ & $99,0(98,9-99,1)$ & $98,4(98,2-98,5)$ & $97,8(97,5-98,1)$ & $98,6(98,4-98,8)$ \\
\hline \multicolumn{7}{|c|}{ Magnésio (mg) } \\
\hline & Adolescentes & $68,9(66,4-71,4)$ & $65,5(63,0-68,1)$ & $66,0(63,3-68,6)$ & $61,2(57,8-64,6)$ & $54,7(50,5-58,8)$ \\
\hline & Adultos & $74,0(72,9-75,1)$ & $70,6(68,9-72,3)$ & $69,7(66,7-72,6)$ & $65,6(62,7-68,6)$ & $58,5(55,4-61,6)$ \\
\hline & Idosos & $77,0(74,2-79,8)$ & $74,8(72,4-77,3)$ & $74,0(72,9-75,2)$ & $69,3(67,0-71,5)$ & $62,8(58,7-66,9)$ \\
\hline \multicolumn{7}{|l|}{ Fósforo (mg) } \\
\hline & Adolescentes & $75,0(71,0-79,0)$ & $72,4(68,0-76,8)$ & $70,7(65,8-75,6)$ & $67,8(64,0-71,6)$ & $69,0(63,0-74,9)$ \\
\hline & Adultos & $14,7(13,4-15,9)$ & $13,3(12,1-14,6)$ & $12,8(11,7-13,9)$ & $11,2(10,2-12,3)$ & $11,7(8,6-14,8)$ \\
\hline & Idosos & $21,6(19,2-24,1)$ & $19,5(16,9-22,1)$ & $18,8(17,9-19,6)$ & $16,5(14,6-18,4)$ & $17,6(13,6-21,7)$ \\
\hline \multicolumn{7}{|l|}{ Cobre (mg) } \\
\hline & Adolescentes & $8,6(7,5-9,7)$ & $4,6(4,3-4,9)$ & $7,2(6,0-8,3)$ & $9,8(8,6-11,1)$ & $3,8(3,2-4,3)$ \\
\hline & Adultos & $13,3(12,0-14,6)$ & $7,3(6,6-8,1)$ & $11,5(10,8-12,1)$ & $15,1(13,7-16,4)$ & $6,2(5,3-7,1)$ \\
\hline & Idosos & $14,3(11,8-16,9)$ & $8,3(6,7-9,9)$ & $12,6(11,0-14,2)$ & $16,3(14,2-18,3)$ & $6,9(5,4-8,4)$ \\
\hline \multicolumn{7}{|l|}{ Zinco (mg) } \\
\hline & Adolescentes & $22,9(18,7-27,1)$ & $23,6(20,5-26,8)$ & $31,8(28,8-34,9)$ & $20,3(19,1-21,4)$ & $14,5(12,0-17,0)$ \\
\hline & Adultos & $18,8(14,3-23,2)$ & $20,3(18,0-22,6)$ & $27,8(25,6-29,9)$ & $17,2(16,3-18,1)$ & $12,0(9,8-14,2)$ \\
\hline & Idosos & $27,3(22,4-32,2)$ & $28,6(25,8-31,4)$ & $37,5(34,5-40,5)$ & $24,4(22,7-26,0)$ & $18,3(15,8-20,9)$ \\
\hline \multicolumn{7}{|c|}{ Vitamina A $(\mathrm{mcg})^{\mathrm{a}}$} \\
\hline & Adolescentes & $85,3(81,5-89,2)$ & $79,4(75,0-83,7)$ & $78,4(76,1-80,6)$ & $73,5(68,2-78,7)$ & $77,8(69,6-86,0)$ \\
\hline & Adultos & $86,9(85,9-87,8)$ & $80,8(79,6-82,1)$ & $79,9(77,2-82,5)$ & $75,6(74,2-77,1)$ & $79,9(74,4-85,3)$ \\
\hline & Idosos & $80,0(77,5-82,5)$ & $73,3(71,1-75,5)$ & $72,6(71,3-74,0)$ & $66,8(64,5-69,1)$ & $72,2(65,0-79,4)$ \\
\hline \multicolumn{7}{|c|}{ Tiamina (mg) } \\
\hline & Adolescentes & $54,2(51,0-57,5)$ & $42,8(40,5-45,1)$ & $38,6(36,8-40,3)$ & $29,2(25,0-33,3)$ & $42,9(41,2-44,5)$ \\
\hline & Adultos & $75,8(73,7-77,9)$ & $65,2(63,3-67,1)$ & $60,6(59,7-61,4)$ & $49,7(45,8-53,6)$ & $65,7(64,4-67,0)$ \\
\hline & Idosos & $79,5(77,3-81,8)$ & $70,5(68,7-72,2)$ & $66,2(65,6-66,9)$ & $54,5(51,4-57,7)$ & $70,5(69,2-71,9)$ \\
\hline \multicolumn{7}{|c|}{ Riboflavina (mg) } \\
\hline & Adolescentes & $50,4(44,5-56,4)$ & $40,8(35,8-45,8)$ & $36,9(30,2-43,5)$ & $36,6(31,1-42,1)$ & $46,6(41,6-51,6)$ \\
\hline & Adultos & $62,2(59,7-64,7)$ & $52,6(51,5-53,6)$ & $48,2(46,0-50,4)$ & $48,3(46,4-50,2)$ & $59,2(58,0-60,4)$ \\
\hline & Idosos & $57,9(53,9-61,9)$ & $47,8(46,0-49,6)$ & $43,9(41,7-46,2)$ & $43,3(42,0-44,6)$ & $54,6(52,5-56,7)$ \\
\hline \multicolumn{7}{|c|}{ Piridoxina (mg) } \\
\hline & Adolescentes & $79,9(76,4-83,4)$ & $76,0(72,4-79,6)$ & $74,7(71,4-78,1)$ & $70,9(66,5-75,3)$ & $81,1(78,2-83,9)$ \\
\hline & Adultos & $93,9(93,2-94,6)$ & $92,2(90,7-93,7)$ & $91,4(90,9-91,9)$ & $89,6(88,3-90,9)$ & $94,8(94,3-95,2)$ \\
\hline & Idosos & $97,9(97,3-98,4)$ & $97,0(96,2-97,9)$ & $96,6(96,2-97,1)$ & $95,4(94,7-96,2)$ & $98,2(97,8-98,5)$ \\
\hline \multicolumn{7}{|c|}{ Cobalamina (mcg) } \\
\hline & Adolescentes & $4,2(1,8-6,6)$ & $10,0(5,5-14,5)$ & $15,7(10,8-20,7)$ & $11,0(6,5-15,5)$ & $7,4(4,0-10,9)$ \\
\hline & Adultos & $6,1(4,6-7,6)$ & $14,8(11,8-17,8)$ & $22,2(21,0-23,5)$ & $15,9(13,5-18,3)$ & $11,1(9,2-13,1)$ \\
\hline & Idosos & $7,9(5,7-10,0)$ & $17,8(13,5-22,1)$ & $26,3(23,7-28,8)$ & $18,8(14,9-22,7)$ & $14,0(10,6-17,3)$ \\
\hline \multicolumn{7}{|c|}{ Vitamina $\mathrm{D}(\mathrm{mcg})^{\mathrm{b}}$} \\
\hline & Adolescentes & $99,8(99,7-100)$ & $99,9(99,9-99,9)$ & $99,9(99,9-100)$ & $99,8(99,8-99,9)$ & $99,9(99,8-99,9)$ \\
\hline & Adultos & $100(99,9-100)$ & $100(100-100)$ & $100(99,9-100)$ & $99,9(99,9-100)$ & $99,9(99,9-100)$ \\
\hline & Idosos & $100(100-100)$ & $100(100-100)$ & $100(100-100)$ & $100(99,9-100)$ & $100(99,9-100)$ \\
\hline \multicolumn{7}{|c|}{ Vitamina $E(m g)^{c}$} \\
\hline & Adolescentes & $89,1(83,8-94,5)$ & $96,0(95,1-97,0)$ & $93,4(91,5-95,3)$ & $94,8(93,5-9,06)$ & $92,3(91,0-93,6)$ \\
\hline & Adultos & $94,1(91,0-97,2)$ & $98,3(98,0-98,7)$ & $96,6(95,6-97,6)$ & $97,5(97,0-97,9)$ & $96,3(95,8-96,9)$ \\
\hline & Idosos & $95,9(92,7-99,2)$ & $98,9(98,6-99,2)$ & $97,9(97,2-98,6)$ & $98,3(97,9-98,6)$ & $97,5(97,0-97,9)$ \\
\hline \multicolumn{7}{|c|}{ Vitamina C (mg) } \\
\hline & Adolescentes & $37,5(32,3-42,7)$ & $30,9(26,1-35,8)$ & $29,2(25,5-32,9)$ & $20,0(17,1-22,9)$ & $28,0(24,7-31,4)$ \\
\hline & Adultos & $48,0(44,0-51,9)$ & $40,8(38,5-43,1)$ & $38,9(37,0-40,8)$ & $27,9(25,4-30,5)$ & $37,6(35,6-39,6)$ \\
\hline & Idosos & $45,2(41,0-49,4)$ & $38,4(36,0-40,9)$ & $36,6(33,4-39,8)$ & $25,5(21,7-29,2)$ & $35,2(32,7-37,7)$ \\
\hline
\end{tabular}

a Equivalente de atividade de retinol (RAE).

${ }^{\mathrm{b}}$ Ergocalciterol (D2) + colecalciterol (D3)

${ }^{c}$ Alfa-tocoferol total. 
à região Norte entre adolescentes e adultos. Além disso, a região Norte despontou com as diferenças mais elevadas nas prevalências de inadequação de vitamina $C$, em contraste com a região Sudeste, entre homens e mulheres de todas as faixas etárias, e de magnésio, em contraste com a região Centro-Oeste, para adultos, mas com prevalência menor de inadequação de cobalamina em relação à região Sul entre mulheres adultas e idosas (Tabelas 9 e 10).

\section{DISCUSSÃO}

Apesar da distância de uma década entre os dois Inquéritos Nacionais de Alimentação, o consumo de energia não variou de forma significativa no período, e os nutrientes que causavam preocupação no primeiro inquérito permanecem sendo aqueles com maiores prevalências de inadequação na segunda investigação, com destaque para cálcio, vitaminas A, D e E, magnésio e piridoxina. Ressalta-se também a alta prevalência de consumo excessivo de sódio nos dois inquéritos, principalmente entre homens adultos.

Os indivíduos mais pobres foram mais vulneráveis ao consumo inadequado da maioria dos nutrientes investigados, com atenção especial para vitaminas A e C, tiamina e riboflavina, que tiveram grande diferença, absoluta e relativa, em comparação com o estrato de maior de renda. Foram observadas disparidades demográficas de acordo com as regiões do país e, de forma geral, as prevalências de inadequação de consumo foram maiores para a região Norte, seguida das regiões Nordeste ou Centro-Oeste.

As prevalências de inadequação aqui apresentadas têm como referência valores ideais para a manutenção de marcadores bioquímicos em indivíduos saudáveis. Elas não indicam, portanto, necessariamente, o aparecimento de desfechos clínicos de saúde. Esses desfechos decorrem de mecanismos metabólicos que envolvem a interação entre a biodisponibilidade e a necessidade de nutrientes, assim como de características individuais relacionadas, por exemplo, a fatores genéticos e ao estado nutricional ao longo da vida ${ }^{18}$.

Poucos estudos com amostra representativa da população brasileira analisaram a prevalência de inadequação da ingestão de nutrientes. Dentre eles, o Estudo de Riscos Cardiovasculares em Adolescentes (Erica) ${ }^{19}$ avaliou adolescentes brasileiros e apontou como nutrientes com maiores prevalências de inadequação os mesmos observados na presente análise, por exemplo o cálcio (prevalência de inadequação de 99\%), vitaminas A (prevalências de inadequação variando entre 60\% e 74\%) e E (prevalência de inadequação de 100\%) e sódio (prevalências de consumo excessivo variando entre $79 \%$ e $91 \%$ ).

A inadequação de $50 \%$ na ingestão de fósforo entre os adolescentes investigados na presente análise também foi observada no estudo Erica, que encontrou prevalências de inadequação ainda mais altas, entre $59 \%$ e $65 \%$. A maior inadequação de fósforo nesta faixa etária se deve ao maior valor de necessidade média de ingestão, aproximadamente o dobro do recomendado para adultos ${ }^{20}$. Destaca-se ainda a importância do fósforo na adolescência, juntamente com o cálcio e a vitamina $\mathrm{D}$, para manutenção adequada do metabolismo ósseo e prevenção de doenças como escoliose e osteoporose em idades mais avançadas ${ }^{21}$.

Estudos em países da Europa, América do Norte, América Latina, África e Ásia também apontam para a ingestão inadequada de nutrientes em diversas populações. Cada região apresenta características específicas em relação aos nutrientes cuja ingestão por sua população é mais crítica. Contudo, em geral, observaram-se altas prevalências de inadequação na ingestão de cálcio, vitaminas A, D, E, zinco, folato e ferro ${ }^{22,23}$.

A evolução da inadequação de nutrientes foi avaliada também com dados de adultos do inquérito norte-americano National Health and Nutrition Examination Survey, de 2003 a 2016. Foi observada redução da inadequação para a maioria dos nutrientes. Ainda assim, cálcio, magnésio, vitaminas A, C, D, e E e folato permaneceram com inadequação acima de $50 \%$ em 2015-2016 24 . 
Cabe destacar, porém, que apesar de diferentes estudos apontarem altas prevalências de inadequação para os mesmos nutrientes, tais resultados precisam ser vistos com cautela, uma vez que as pesquisas diferem nos métodos de avaliação dietética, nos valores de referência e nas análises utilizadas para estimar o consumo usual.

Alguns aspectos importantes sobre a alta prevalência de inadequação da ingestão de cálcio e vitamina $\mathrm{D}$ precisam ser apontados. Os valores de referência desses dois nutrientes foram revistos na última década, com base na melhor evidência disponível à época ${ }^{20}$. No entanto, assumiu-se, para estabelecer esses valores, pouca ou nenhuma exposição solar ${ }^{20}$, o que pode não ser aplicável em países de clima predominantemente tropical, como o Brasil. Parte da necessidade de vitamina $D$ pode ser atendida por síntese a partir da exposição solar. Assim, em estudo realizado no município de São Paulo, quase 100\% da população tinha ingestão inadequada de vitamina $\mathrm{D}$, mas aproximadamente metade das pessoas apresentavam nível sérico adequado ${ }^{25}$.

As necessidades de cálcio também são fonte de debate intenso, que envolve tanto a adequação dos estudos de balanço quanto a não confirmação dos efeitos benéficos para saúde óssea em metanálises de estudos longitudinais ${ }^{26}$. No entanto, apesar das críticas aos valores de referência, é importante salientar que a frequência do consumo de alimentos fonte de cálcio e vitamina D, como laticínios e pescados, é muito baixa desde o INA 2008-2009 ${ }^{10}$.

O consumo excessivo e inadequado de sódio se manteve entre os dois inquéritos, resultado alinhado com a Pesquisa Nacional de Saúde de 2013, que estimou o consumo de sal na população brasileira pela excreção urinária de sódio. O consumo verificado, maior entre os homens, foi o dobro da quantidade máxima de ingestão recomendada pela Organização Mundial da Saúde ${ }^{27}$.

A alta prevalência do consumo excessivo de sódio é uma preocupação de décadas no Brasil $^{28}$ e no mundo ${ }^{29}$. O seu consumo em excesso, associado a uma dieta pobre em potássio, aumenta os níveis pressóricos e representa risco para o desenvolvimento da hipertensão arterial, que por sua vez tem sido elencada como um dos fatores de risco mais importantes para morbimortalidade por doenças cardiovasculares ${ }^{30}$. Outro resultado de destaque foi o aumento, entre idosos, da inadequação de riboflavina, vitamina que pode exercer efeitos neuroprotetores para alguns distúrbios neurológicos (por exemplo, doença de Parkinson, enxaqueca e esclerose múltipla), tendo como principais fontes o leite e produtos lácteos, vísceras e carne bovina ${ }^{31}$.

Indivíduos com menor renda domiciliar per capita foram aqueles com maiores prevalências de inadequação para a maioria dos nutrientes investigados. De forma similar, em geral, regiões mais pobres do país apresentaram maiores prevalências de inadequação. Estudos com a população norte-americana também mostraram que, entre adultos de baixo nível socioeconômico, aumentava a proporção de indivíduos com ingestão inadequada de nutrientes ${ }^{24}$, enquanto nos maiores níveis de renda o risco de inadequação diminuía ${ }^{32}$.

As disparidades socioeconômicas e demográficas aqui apontadas já foram observadas em outras análises do inquérito de 2008-2009 ${ }^{33}$ e em outras amostras de base populacional brasileira ${ }^{34}$. Essas disparidades também foram observadas na avaliação do consumo de alimentos usando a mesma base de dados, verificando-se redução na frequência do consumo de frutas entre 2008-2009 e 2017-2018, mais acentuada no quarto de renda mais baixo. Em contrapartida, os resultados de 2017-2018 apontaram que alimentos tipicamente brasileiros, como arroz, feijão, farinha de mandioca, milho e alimentos à base de milho foram consumidos com mais frequência entre indivíduos de menor renda ${ }^{10}$.

Diferenças regionais acompanham as diferenças de renda. Alimentos como farinha de mandioca, milho e alimentos à base de milho foram mais frequentemente consumidos em regiões mais pobres, como Norte e Nordeste, respectivamente. Por outro lado, as regiões Sul e Sudeste apresentaram as maiores médias de consumo per capita de frutas e verduras ${ }^{10}$. Destaca-se ainda que os resultados da POF 2017--2018 mostraram também 
como o acesso regular e permanente dos domicílios a alimentos de qualidade e em quantidade suficiente é desigual no Brasil. As regiões Norte e Nordeste apresentaram as menores proporções de domicílios particulares em situação de segurança alimentar. Menos da metade dos moradores dessas regiões tiveram acesso pleno e regular aos alimentos ${ }^{5}$. Portanto, qualquer estratégia que objetive melhorar a ingestão de nutrientes no Brasil precisa enfatizar o acesso à alimentação adequada aos indivíduos mais desfavorecidos e residentes nas regiões mais pobres.

O presente estudo apresenta algumas limitações. Primeiro, a ingestão de nutrientes não considerou as informações sobre uso de suplementos nutricionais aplicadas no último inquérito. Os indivíduos foram questionados somente sobre o uso nos últimos 30 dias, mas sem informações sobre quantidade e frequência consumida. Logo, não foi possível estimar o quantitativo de nutrientes fornecidos por suplementos. O uso de multivitamínicos, vitaminas do complexo B e vitamina $\mathrm{C}$ foi relatado por 11,1\% da população, variando até $19,5 \%$ em idosas, e o uso de suplemento de cálcio foi relatado por 4,6\%, variando até $21,3 \%$ também em idosas.

A segunda limitação foi a mudança no método de coleta do consumo alimentar, que dificulta a comparação entre os dois inquéritos. A mudança se deu porque estudos de validação sistematicamente mostraram que a coleta por meio de R24h assistida por computador diminui o sub-relato de energia, sódio, potássio e proteína ${ }^{35}$. Assim, acredita-se que a alteração tenha permitido estimativas mais acuradas. Entretanto, o sub-relato na ingestão energética, comum na coleta por R24h, provavelmente levou a prevalências de inadequação superestimadas. Mas como a ingestão calórica foi semelhante entre os dois inquéritos (1.753 kcal em 2008-2009 e 1.748 kcal em 2017-2018), é provável que o efeito desse possível subrelato tenha sido semelhante.

As elevadas e persistentes prevalências de inadequação verificadas neste estudo apontam para uma alimentação nutricionalmente deficiente, em um contexto de sindemia global de obesidade, desnutrição e mudanças climáticas ${ }^{36}$. Tendo em vista esse cenário, a FAO reconhece o desafio de combater a má nutrição em todas as suas formas, incluindo a desnutrição, carências nutricionais específicas, sobrepeso, obesidade e DCNT associadas à alimentação ${ }^{37}$.

No Brasil, o acesso à alimentação adequada como direito está previsto em lei ${ }^{38} \mathrm{e} \mathrm{incorporado}$ à Constituição Federal ${ }^{39}$. Nessa perspectiva, o acesso físico e econômico aos alimentos deve garantir não somente alimentação em quantidade suficiente como nutricionalmente adequada. No entanto, como demonstrou o presente artigo, a ingestão de nutrientes tem se mostrado inadequada entre os brasileiros.

\section{CONCLUSÃO}

Prevalências elevadas de inadequação de ingestão de nutrientes e consumo excessivo de sódio foram verificadas em ambos os inquéritos. A prevalência de inadequação se modifica de acordo com as variáveis sociodemográficas, aumentando entre indivíduos nos menores estratos de renda e nas regiões mais pobres do país.

\section{REFERÊNCIAS}

1. Afshin A, Sur PJ, Fay KA, Cornaby L, Ferrara G, Salama JS, et al; GBD 2017 Diet Collaborators. Health effects of dietary risks in 195 countries, 1990-2017: a systematic analysis for the Global Burden of Disease Study 2017. Lancet. 2019;393(10184):1958-72. https://doi.org/10.1016/S0140-6736(19)30041-8

2. Food and Agriculture Organization; International Fund for Agricultural Development; United Nations International Children's Emergency Fund; World Food Programme; World Health Organization. The State of Food Security and Nutrition in the World, 2020: transforming food systems for affordable healthy diets. Rome (IT): FAO; 2020. 
3. United Nations, Department of Economic and Social Affairs. The Millennium Development Goals Report 2015. New York: UN; 2015 [citado 9 mar 2021]. Disponível em: https://www.un.org/development/desa/publications/mdg-report-2015.html

4. Instituto Brasileiro de Geografia e Estatística, Diretoria de Pesquisas, Coordenação de Trabalho e Rendimento. Pesquisa Nacional de Saúde, 2019: atenção primária à saúde e informações antropométricas: Brasil. Rio de Janeiro: IBGE; 2020 [citado 29 out 2020]. Disponível em: https://biblioteca.ibge.gov.br/visualizacao/livros/liv101758.pdf

5. Instituto Brasileiro de Geografia e Estatística, Diretoria de Pesquisas, Coordenação de Trabalho e Rendimento. Pesquisa de Orçamentos Familiares 2017-2018: análise da segurança alimentar no Brasil. Rio de Janeiro: IBGE; 2020 [citado 29 out 2020]. Disponível em: https://biblioteca.ibge.gov.br/visualizacao/livros/liv101749.pdf

6. Segal L, Opie RS. A nutrition strategy to reduce the burden of diet related disease: access to dietician services must complement population health approaches. Front Pharmacol. 2015;6:160. https://doi.org/10.3389/fphar.2015.00160

7. Bourre JM. Effects of nutrients (in food) on the structure and function of the nervous system: update on dietary requirements for brain. Part 1: micronutrients. J Nutr Health Aging. 2006;10(5):377-85.

8. Tardy AL, Pouteau E, Marquez D, Yilmaz C, Scholey A. Vitamins and minerals for energy, fatigue and cognition: a narrative review of the biochemical and clinical evidence. Nutrients. 2020;12(1):228. https://doi.org/10.3390/nu12010228

9. European Food Safety Authority. Panel on Dietetic Products, Nutrition, and Allergies (NDA). Scientific opinion on principles for deriving and applying Dietary Reference Values. EFSA J. 2010;8(3):1458. https://doi.org/10.2903/j.efsa.2010.1458

10. Instituto Brasileiro de Geografia e Estatística, Diretoria de Pesquisas, Coordenação de Trabalho e Rendimento. Pesquisa de Orçamentos Familiares 2017-2018: análise do consumo alimentar pessoal no Brasil. Rio de Janeiro: IBGE; 2020 [citado 29 out 2020]. Disponível em: https://biblioteca.ibge.gov.br/visualizacao/livros/liv101742.pdf

11. Instituto Brasileiro de Geografia e Estatística, Diretoria de Pesquisas, Coordenação de Trabalho e Rendimento. Pesquisa de Orçamentos Familiares 2008-2009: análise do consumo alimentar pessoal no Brasil. Rio de Janeiro: IBGE; 2011 [citado 29 out 2020]. Disponível em: https://biblioteca.ibge.gov.br/visualizacao/livros/liv50063.pdf

12. Moshfegh AJ, Rhodes DG, Baer DJ, Murayi T, Clemens JC, Rumpler WV, et al. The US Department of Agriculture Automated Multiple-Pass Method reduces bias in the collection of energy intakes. Am J Clin Nutr. 2008;88(2):324-32. https://doi.org/10.1093/ajcn/88.2.324

13. Tabela Brasileira de Composição de Alimentos (TBCA). Versão 7.1. São Paulo: Universidade de São Paulo, Food Research Center; 2020 [citado 29 out 2020]. Disponível em: http://www.tbca.net.br

14. International Network of Food Data Systems. Standards and guidelines. Rome (IT): FAO; 2019 [citado 29 out 2020]. Disponível em: http://www.fao.org/infoods/infoods/standards-guidelines/en/

15. Tooze JA, Midthune D, Dodd KW, Freedman LS, Krebs-Smith SM, Subar AF, et al. A new statistical method for estimating the usual intake of episodically consumed foods with application to their distribution. J Am Diet Assoc. 2006;106(10):1575-87. https://doi.org/10.1016/j.jada.2006.07.003

16. Institute of Medicine (US), Subcommittee on Interpretation and Uses of Dietary Reference Intakes. Dietary Reference Intake: applications in dietary assessment. Washington, DC: National Academies Press; 2000. https://doi.org/10.17226/9956

17. National Academies of Sciences, Engineering, and Medicine; Health and Medicine Division; Food and Nutrition Board; Committee to Review the Dietary Reference Intakes for Sodium and Potassium. Dietary reference intakes for sodium and potassium. Washington, DC: National Academies Press; 2019. https://doi.org/10.17226/25353

18. Stover PJ, Garza C, Durga J, Field MS. Emerging concepts in nutrient needs. J Nutr. 2020;150 Suppl 1:2593S-601S. https://doi.org/10.1093/jn/nxaa117

19. Souza AM, Barufaldi LA, Abreu GA, Giannini DT, Oliveira CL, Santos MM, et al. ERICA: intake of macro and micronutrients of Brazilian adolescents. Rev Saude Publica. 2016;50 Suppl 1. https://doi.org/10.1590/s01518-8787.2016050006698

20. Institute of Medicine (US), Standing Committee on the Scientific Evaluation of Dietary Reference Intakes. Dietary reference intakes for calcium, phosphorus, magnesium, vitamin d, and fluoride. Washington (DC): National Academies Press; 1997. https://doi.org/10.17226/5776 
21. Batista RMBF, Martins DE, Wajchenberg M, Lazaretti M, Puertas EB, Hayashi LF. Association between vitamin d levels and adolescent idiopathic scoliosis. Coluna/Columna. 2014;13(4):275-8. https://doi.org/10.1590/S1808-18512014130400432

22. Roman Viñas B, Ribas Barba L, Ngo J, Gurinovic M, Novakovic R, Cavelaars A, et al. Projected prevalence of inadequate nutrient intakes in Europe. Ann Nutr Metab. 2011;59(2-4):84-95. https://doi.org/10.1159/000332762

23. Beal T, Massiot E, Arsenault JE, Smith MR, Hijmans RJ. Global trends in dietary micronutrient supplies and estimated prevalence of inadequate intakes. PLoS One. 2017;12(4):e0175554. https://doi.org/10.1371/journal.pone.0175554

24. Han S, Wu L, Wang W, Li N, Wu X. Trends in dietary nutrients by demographic characteristics and BMI among US adults, 2003-2016. Nutrients. 2019;11(11):2617. https://doi.org/10.3390/nu11112617

25. Martini LA, Verly Jr E, Marchioni DM, Fisberg RM. Prevalence and correlates of calcium and vitamin D status adequacy in adolescents, adults, and elderly from the Health Survey-São Paulo. Nutrition. 2013;29(6):845-50. https://doi.org/10.1016/j.nut.2012.12.009

26. Willett WC, Ludwig DS. Milk and health. N Engl J Med. 2020;382(7):644-54. https://doi.org/10.1056/nejmra1903547

27. Mill JG, Malta DC, Machado IE, Pate A, Pereira CA, Jaime PC, et al. Estimativa do consumo de sal pela população brasileira: resultado da Pesquisa Nacional de Saúde 2013. Rev Bras Epidemiol. 2019;22 Supl 2:E190009.Supl.2. https://doi.org/10.1590/1980-549720190009.supl.2

28. Malta DC, Morais Neto OL, Silva Junior JB. Apresentação do Plano de Ações Estratégicas para o Enfrentamento das Doenças Crônicas não Transmissíveis no Brasil, 2011 a 2022. Epidemiol Serv Saude. 2011;20(4):425-38. https://doi.org/10.5123/S1679-49742011000400002

29. World Health Organization. Guideline: sodium intake for adults and children. Geneva (CH): WHO; 2012 [citado 29 out 2020]. Disponível em: https://apps.who.int/iris/ handle/10665/77985

30. O'Donnell MJ, Xavier D, Liu L, Zhang H, Chin SL, Rao-Melacini P, et al. Risk factors for ischaemic and intracerebral haemorrhagic stroke in 22 countries (the INTERSTROKE study): a case-control study. Lancet. 2010;376(9735):112-23. https://doi.org/10.1016/s0140-6736(10)60834-3

31. Saedisomeolia A, Ashoori M. Riboflavin in human health: a review of current evidences. Adv Food Nutr Res. 2018;83:57-81. https://doi.org/10.1016/bs.afnr.2017.11.002

32. Bailey RL, Akabas SR, Paxson EE, Thuppal SV, Saklani S, Tucker KL. Total usual intake of shortfall nutrients varies with poverty among US adults. J Nutr Educ Behav. 2017;49(8):639-46.e3. https://doi.org/10.1016/j.jneb.2016.11.008

33. Araujo MC, Verly Junior E, Junger WL, Sichieri R. Independent associations of income and education with nutrient intakes in Brazilian adults: 2008-2009 National Dietary Survey. Public Health Nutr. 2014;17(12):2740-52. https://doi.org/10.1017/s1368980013003005

34. Verly Junior E, Cesar CLG, Fisberg RM, Marchioni DM. Socio-economic variables influence the prevalence of inadequate nutrient intake in Brazilian adolescents: results from a population-based survey. Public Health Nutr. 2011;14(9):1533-8. https://doi.org/10.1017/s1368980011000760

35. Park Y, Dodd KW, Kipnis V, Thompson FE, Potischman N, Schoeller DA, et al. Comparison of self-reported dietary intakes from the Automated Self-Administered 24-h recall, 4-d food records, and food-frequency questionnaires against recovery biomarkers. Am J Clin Nutr. 2018;107(1):80-93. https://doi.org/10.1093/ajcn/nqx002

36. Swinburn BA, Kraak VI, Allender S, Atkins VJ, Baker PI, Bogard JR, et al. The Global Syndemic of Obesity, Undernutrition, and Climate Change: The Lancet Commission report. Lancet. 2019;393(10173):791-846. https://doi.org/10.1016/S0140-6736(18)32822-8

37. High Level Panel of Experts, Committee on World Food Security. Investing in smallholder agriculture for food security: a report by the High Level Panel of Experts on Food Security and Nutrition. Rome (IT): HLPE; 2013 [citado 29 out 2020]. Disponível em: http://www.fao.org/3/a-i2953e.pdf 
38. Brasil. Lei № 11.346, de 15 de setembro de 2006. Cria o Sistema Nacional de Segurança Alimentar e Nutricional - SISAN com vistas em assegurar o direito humano à alimentação adequada e dá outras providências. Brasília, DF; 2006 [citado 9 mar 2021]. Disponível em: http://www.planalto.gov.br/ccivil_03/_ato2004-2006/2006/lei/111346.htm

39. Brasil. Emenda Constitucional, Nº 64, de 4 de fevereiro de 2010.

Altera o art. $6^{\circ}$ da Constituição Federal, para introduzir a alimentação como direito social. Brasília, DF; 2010 [citado 9 mar 2021]. Disponível em: www.planalto.gov.br/ccivil_03/ constituicao/emendas/emc/emc64.htm

Financiamento: Conselho Nacional de Desenvolvimento Científico e Tecnológico (CNPq/Ministério da Saúde - Processo 443369/2016-0).

Contribuição dos Autores: Concepção e planejamento do estudo: EVJ, DMLM, MCA, EC, EMY, RS, RAP. Análise e interpretação dos dados: EVJ, DMLM, MCA, EC, DCRSO. Elaboração e revisão do manuscrito: EVJ, DMLM, MCA, EC, DCRSO. Aprovação da versão final: EVJ, DMLM, MCA, EC, DCRSO, EMY, RS, RAP. Responsabilidade pública pelo conteúdo do artigo: EVJ, DMLM, MCA, EC, DCRSO, EMY, RS, RAP.

Conflito de Interesses: Os autores declaram não haver conflito de interesses. 